\title{
Article \\ Artemether-Loaded Zein Nanoparticles: An Innovative Intravenous Dosage Form for the Management of Severe Malaria
}

\author{
Yaa Boateng-Marfo ${ }^{1,2,3}$, Yuancai Dong ${ }^{1}$, Wai Kiong $\mathrm{Ng}^{1,2, *}$ and Hai-Shu Lin ${ }^{2,4, *(D)}$ \\ 1 Institute of Chemical and Engineering Sciences, Agency for Science, Technology and Research, 1 Pesek Road, \\ Jurong Island, Singapore 627833, Singapore; nayabdear@gmail.com (Y.B.-M.); dongyuancai@gmail.com (Y.D.) \\ 2 Department of Pharmacy, National University of Singapore, 18 Science Drive 4, Singapore 117543, Singapore \\ 3 Department of Pharmaceutical Sciences, Sunyani Technical University, P.O. Box 206 Sunyani, Ghana \\ 4 College of Pharmacy, Shenzhen Technology University, 3002 Lantian Road, Pingshan District, \\ Shenzhen 518118, China \\ * Correspondence: ng_wai_kiong@ices.a-star.edu.sg (W.K.N.); linhaishu@sztu.edu.cn (H.-S.L.)
}

check for updates

Citation: Boateng-Marfo, Y.; Dong,

Y.; Ng, W.K.; Lin, H.-S.

Artemether-Loaded Zein Nanoparticles: An Innovative Intravenous Dosage Form for the Management of Severe Malaria. Int. J. Mol. Sci. 2021, 22, 1141. https:// doi.org/10.3390/ijms22031141

Academic Editor: Maciej Jarzębski

Received: 4 January 2021

Accepted: 17 January 2021

Published: 24 January 2021

Publisher's Note: MDPI stays neutral with regard to jurisdictional claims in published maps and institutional affiliations.

Copyright: (c) 2021 by the authors. Licensee MDPI, Basel, Switzerland. This article is an open access article distributed under the terms and conditions of the Creative Commons Attribution (CC BY) license (https:// creativecommons.org/licenses/by/ $4.0 /)$.

\begin{abstract}
Artemether, an artemisinin derivative, is used in the management of life-threatening severe malaria. This study aimed to develop an intravenous dosage form of artemether using nanotechnology. Artemether-loaded zein nanoparticles were prepared by modified antisolvent precipitation using sodium caseinate as a stabilizer. Subsequently, the physicochemical properties of the nanoparticles were characterized; the in vitro hemolytic property was examined with red blood cells, while the pharmacokinetic profile was evaluated in Sprague-Dawley rats after intravenous administration. The artemether-loaded zein nanoparticles were found to display good encapsulation efficiency, excellent physical stability and offer an in vitro extended-release property. Interestingly, encapsulation of artemether into zein nanoparticles substantially suppressed hemolysis, a common clinical phenomenon occurring after artemisinin-based antimalarial therapy. Upon intravenous administration, artemether-loaded zein nanoparticles extended the mean residence time of artemether by $\sim 80 \%$ in comparison to the free artemether formulation $(82.9 \pm 15.2$ versus $45.6 \pm 16.4 \mathrm{~min}$, $p<0.01$ ), suggesting that the nanoparticles may prolong the therapeutic duration and reduce the dosing frequency in a clinical setting. In conclusion, intravenous delivery of artemether by artemetherloaded zein nanoparticles appears to be a promising therapeutic option for severe malaria.
\end{abstract}

Keywords: artemether; artemisinin; extended release; hemolysis; intravenous; nanoparticles; pharmacokinetics; severe malaria; sodium caseinate; zein

\section{Introduction}

Malaria is a life-threatening tropical disease caused by Plasmodium parasites that are transmitted to people through the bites of infected female Anopheles mosquitoes [1-3]. According to World Malaria Report 2020, there were 229 million cases worldwide and more than 400,000 people died of malaria in 2019 [3]. Children under the age of five are the most vulnerable group affected by malaria and they accounted for $67 \%$ of global malaria deaths [3]. It is generally believed that malaria is associated with poverty and sometimes it is even the cause of poverty $[4,5]$.

The management of malaria has been revolutionarily improved by the introduction of the artemisinin derivatives in the 1990s, a group of semisynthetic compounds produced from artemisinin (Figure 1a), a sesquiterpene lactone endoperoxide originally isolated from the traditional Chinese herb Artemisia annua [1,6]. As artemisinin derivatives are rapidly effective, safe and well tolerated, artemisinin-based combination treatments are recommended by the World Health Organization (WHO) as first-line therapies in all malaria endemic countries [1,7]. The discovery of artemisinin has saved millions of lives 
and was acknowledged by the award of the Nobel Prize in Physiology or Medicine to Professor Youyou Tu in 2015 [1].

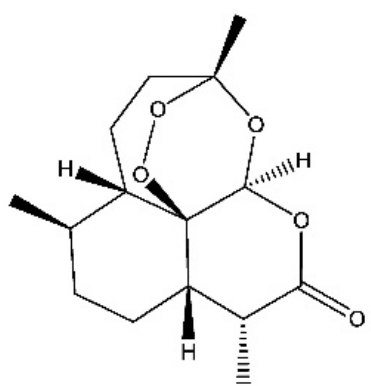

(a)

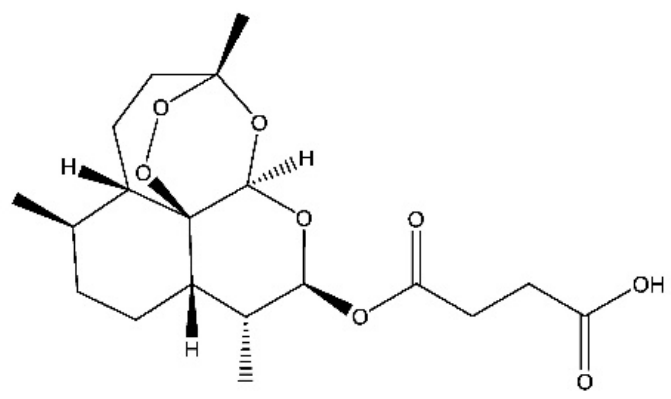

(b)

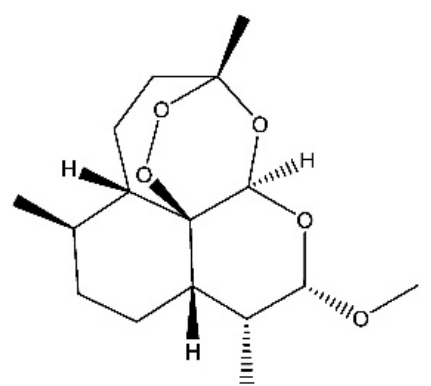

(c)

Figure 1. Chemical structures of artemisinin (a), artesunate (b) and artemether (c).

As the mortality from untreated severe malaria can be as high as $100 \%$, severe malaria is a medical emergency, and requires intensive nursing care and careful management $[2,7]$. Although artemisinin derivatives such as artesunate (Figure 1b) and artemether (Figure 1c) can be given through oral administration to treat uncomplicated malaria [7], intravenous or intramuscular administration of artesunate is the first choice for the management of severe malaria [2,7]. In case injectable artesunate is not available, parenteral artemether is an alternative option [7]. Different from artesunate, artemether displays limited aqueous solubility and its parenteral formulation is only available as a premixed oil-based solution for intramuscular injection [8]. When dosed intramuscularly, artemether may be absorbed slowly but erratically, resulting in a smaller survival benefit than parenteral artesunate $[2,7]$. Clearly, it of great clinical significance to formulate artemether into an intravenous dosage form for the management of severe malaria.

During the past two decades, advances in nanotechnology have made great contributions to pharmaceutical industries. The formulation development of paclitaxel, a water insoluble chemotherapeutic agent with broad-spectrum activity in many solid tumors is an excellent example [9]. Because of the solubility issue, paclitaxel is formulated into parenteral dosage form with polyethoxylated castor oil (Cremophor ${ }^{\circledR}$ EL) and ethanol in the conventional formulation (Taxol ${ }^{\circledR}$; CrEL-paclitaxel) [9]. However, due to vehicle toxicity, this formulation is commonly associated with adverse effects such as hypersensitivity reactions, neutropenia and neuropathy [9]. Abraxane ${ }^{\circledR}$, the $130 \mathrm{~nm}$ human serum albumin-bound paclitaxel (nab-paclitaxel), is the first parenteral nanoparticle formulation approved for clinical application in medical history [9]. Since Abraxane ${ }^{\circledR}$ selectively delivers larger amounts of paclitaxel to tumors while avoiding some of the solvent related toxicities of the conventional formulation, it displays clinical efficacy and safety superior to the conventional formulation [9]. Clearly, protein-based nanoparticles are a practical strategy for parenteral delivery of water insoluble therapeutic agents.

In our recent study, artemether was formulated into an intravenous dosage form using nanoparticle albumin bound technology [10]. Such a formulation not only enhanced the dissolution of artemether, but also decreased hemolysis [10], a common clinical phenomenon occurred after artemisinin-based therapy [11]. However, as malaria is a disease of poverty [4,5], excipients with higher affordability are more appropriate for the intravenous delivery of antimalaria agents. Zein, a plant protein obtained from corn, has attracted significant interest in the biomedical community over the past decade [12,13]. As it is biodegradable, biocompatible and cost-effective, zein appears to be a promising excipient $[12,13]$. In the present study, artemether-loaded zein nanoparticles were prepared by modified antisolvent precipitation using sodium caseinate as a stabilizer. Subsequently, their physicochemical properties were characterized; the in vitro hemolytic property was examined with fresh red blood cells, while the pharmacokinetic profile was evaluated in 
Sprague-Dawley rats after intravenous administration. Hopefully, the information obtained from this study will facilitate the development of an innovative therapeutic option for severe malaria.

\section{Results}

\subsection{Preparation of Artemether-Load Zein Nanoparticles}

As displayed in Figure 2, artemether-load zein nanoparticles were prepared by modified antisolvent precipitation using sodium caseinate as a stabilizer. When aqueous sodium caseinate solution was added to the ethanol solution with artemether and zein, ethanol content reduced sharply, providing a constant microenvironment for the formation of nanoparticles with more ordered structural arrangements of component molecules. It also led to the production of nanoparticles with monomodal distribution and narrow particle size distribution [14]. On the contrary, dropwise addition of ethanol solution to aqueous solution results in particles with a less ordered structural arrangement and multimodal particle size distribution. It also causes increased exposure of the hydrophobic amino acids in zein molecules and therefore requires more stabilizer in order to stabilize the nanoparticles [14].

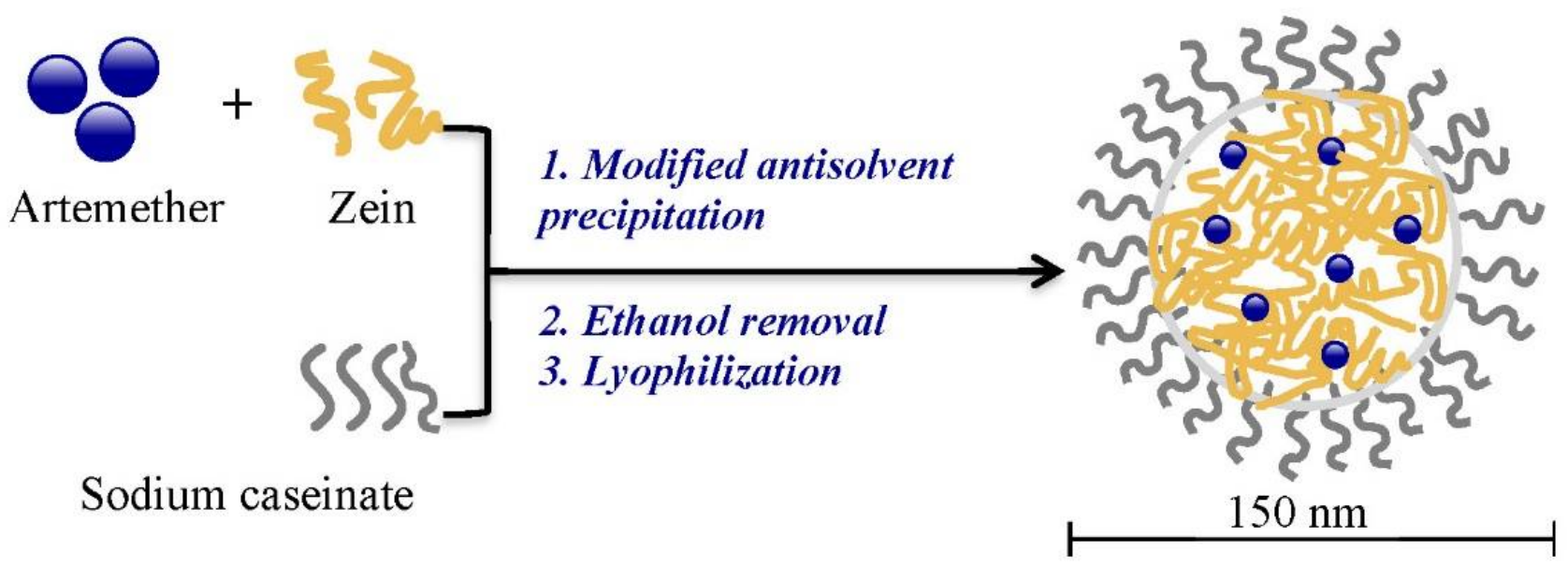

Figure 2. Preparation of artemether-loaded zein nanoparticles.

\subsection{Optimization of Formulation and Process Parameters}

\subsubsection{Sodium Caseinate/Zein Mass Ratio}

Sodium caseinate serves as a stabilizer and cryoprotectant in this work. It also enhances the cellular uptake of nanoparticles [15]. Sodium caseinate is amphiphilic and charged in nature. It adsorbs to the surface of nanoparticles and stabilizes them by both electrostatic repulsion and steric hindrance-i.e., electrosteric stabilization $[16,17]$. As shown in Figure 3, zeta potential shifted from positive values to negative values as the sodium caseinate/zein concentration increased. This is evidence of stabilization by electrostatic repulsion between nanoparticles. Moreover, a further decrease in particle size occurred by increasing the sodium caseinate/zein ratio above 0.75:1 (Figure 4a). Since the zeta potential was higher than $-30 \mathrm{mV}$ at a sodium caseinate/zein ratio of 0.75:1, the decrease in particle size with a higher sodium caseinate/zein ratio is suggestive of stabilization by steric hindrance. 


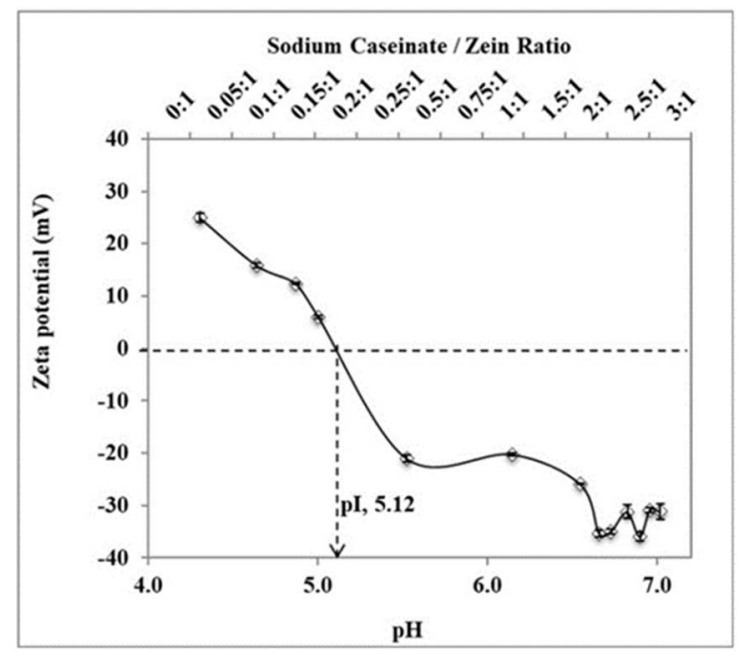

Figure 3. A plot of zeta potential against $\mathrm{pH}$ as sodium caseinate/zein ratio varied from $0: 1$ to 3:1. Zeta potential shifted from positive values to negative values as sodium caseinate/zein ratio increased. Isoelectric point ( $\mathrm{pI}, \mathrm{pH}$ at which zeta potential is zero) was extrapolated to be 5.12. The experimental data were obtained from the nanoparticles prepared from three different batches $(n=3)$; symbols represent mean values while error bars represent standard deviation (SD).
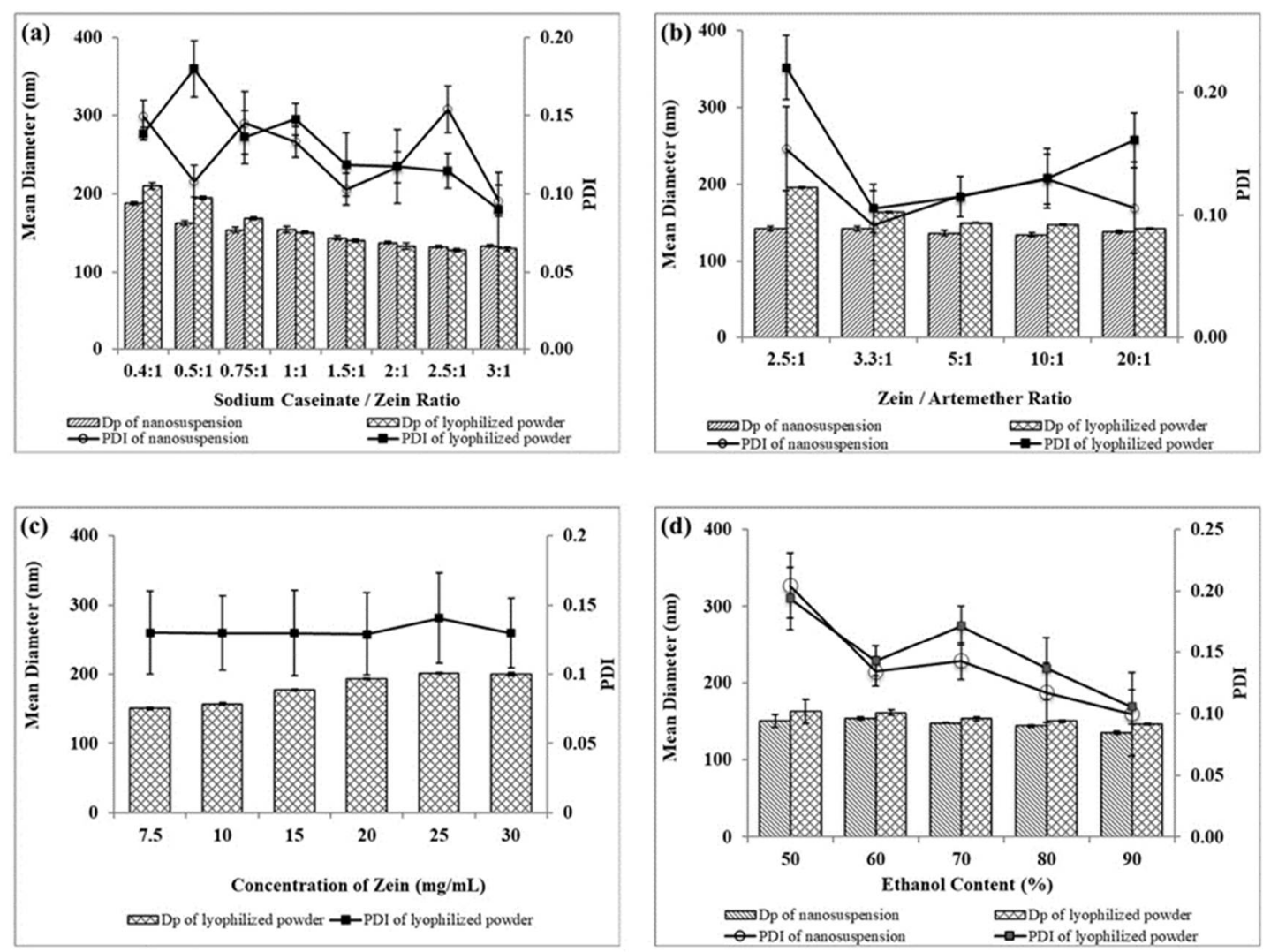

Figure 4. Effects of formulation variables (a) sodium caseinate/zein ratio, (b) zein/artemether ratio, (c) concentration of zein and (d) ethanol content on particle size and polydispersity index (PDI) of artemether-loaded zein nanoparticles in nanosuspension and lyophilized powder forms. The experimental data were obtained from the nanoparticles prepared from three different batches $(n=3)$; symbols represent mean values while error bars represent SD.

The effects of the sodium caseinate/zein ratio on the size of artemether-loaded zein nanoparticles were studied by varying the sodium caseinate/zein ratio from 0:1 to 3:1. The physical appearance of artemether-loaded zein nanosuspension (before ethanol removal) 
prepared using a sodium caseinate/zein ratio from 0:1 to 0.25:1 is shown in Figure 5. At a caseinate/zein ratio of $0: 1$ to $0.1: 1$, relatively larger particles were formed. When the sodium caseinate/zein ratio was increased to $0.15: 1$, an extensive coagulation of zein occurred forming a big yellow lump in a clear medium. This is due to the high positive charge of zein, which dominated the system at a sodium caseinate/zein ratio of 0:1 to $0.1: 1$, with zeta potential values of +25 to $+16 \mathrm{mV}$. As the sodium caseinate/zein ratio increased to $0.15: 1$, there was an extensive neutralization of the positive charge of zein giving a zeta potential value of $+6 \mathrm{mV}$ and $\mathrm{pH}$ of 5 . From a plot of zeta potential against $\mathrm{pH}$ (Figure 3), the $x$-intercept was extrapolated to be 5.12, representative of isoelectric point (pI) of the system. Zein has a high tendency to aggregate in water because its original pI of 6.2 is closer to neutral pH [18]. The presence of sodium caseinate (pI: 4.5) [19] shifted the $\mathrm{pI}$ of the artemether-loaded zein nanoparticles further away from neutral $\mathrm{pH}$ to 5.12, reducing the tendency of the nanoparticles to aggregate in aqueous environments. The lack of charge on the artemether-loaded zein nanoparticles at the $\mathrm{pI}$ is responsible for the extensive coagulation of zein, forming a lump a clear medium. Further increase in sodium caseinate/zein ratio above 0.15:1 led to a shift in zeta potential to negative values, which conferred stability on the nanoparticles by minimizing aggregation. Though artemetherloaded zein nanoparticles prepared at a sodium caseinate/zein ratio of $0.25: 1$ were stable with a zeta potential value of $-20 \mathrm{mV}$, nanoparticles prepared at sodium caseinate/zein ratio below 0.4:1 were not redispersible after lyophilization. This indicated that a higher caseinate/zein proportion was required for cryoprotection during lyophilization.

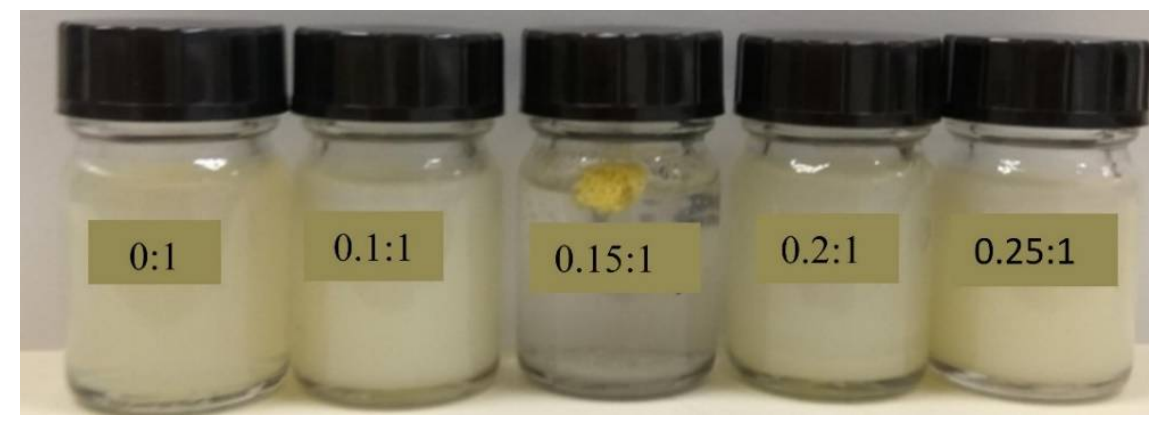

Figure 5. Physical appearances of artemether-loaded zein nanosuspension (before ethanol removal) prepared using sodium caseinate/zein ratio from 0:1 to 0.25:1.

A sharp decrease in particle size from $210 \pm 4$ to $150 \pm 2 \mathrm{~nm}$ was observed as the sodium caseinate/zein ratio increased from 0.4:1 to 1:1. This was followed by a gradual decrease to $129 \pm 2 \mathrm{~nm}$ as the sodium caseinate/zein ratio increased to 3:1, as shown in Figure 4a. Clearly, a sodium caseinate/zein ratio $\geq 0.4: 1$ was sufficient for cryoprotection. Therefore, there was no significant difference between the particle size of the nanosuspensions and that of the lyophilized powders. Particle size decreased with an increasing sodium caseinate/zein ratio because higher sodium caseinate proportions increased their stabilizing effect on the nanoparticles. Varying the sodium caseinate/zein ratio from 0.4:1 to 3:1 did not significantly affect the polydispersity index (PDI) in any order. PDI values between 0.10 and 0.18 , as presented in Figure $4 a$, are indicative of particles with a narrow size distribution. Sodium caseinate/zein ratio 1:1 was selected for the subsequent optimization steps.

\subsubsection{Zein/Artemether Mass Ratio}

The impacts of the zein/artemether ratio on size and PDI of artemether-loaded zein nanoparticles are presented in Figure $4 \mathrm{~b}$. When the zein/artemether ratio increased, the proportion of the drug decreased, resulting in smaller particles. This was evident in the decrease in particle size from $196 \pm 1$ to $142 \pm 3 \mathrm{~nm}$ as the zein/artemether ratio was increased from of 2.5:1 to 20:1. Nanoparticles prepared without artemether exhibited a size of $139 \pm 1 \mathrm{~nm}$. This was probably due to the fact that as the zein/artemether ratio increased, 
the same amount of zein incorporated in the decreasing amount of artemether. In addition, decreasing the proportion of artemether increased the stabilizing effect of sodium caseinate on nanoparticles resulting in less aggregation. The PDI of nanoparticles fell between 0.09 \pm 0.03 and $0.22 \pm 0.03$ and did not follow any particular order. A zein/artemether ratio of $5: 1$ was used for the subsequent optimization steps.

\subsubsection{Zein Concentration}

The impact from zein concentration on particle size was examined by varying it from 7.5 to $30 \mathrm{mg} / \mathrm{mL}$, while keeping the zein/artemether ratio and sodium caseinate constant. As shown in Figure 4c), when the zein concentration increased from 7.5 to $25 \mathrm{mg} / \mathrm{mL}$, particle size increased from $150 \pm 2$ to $201 \pm 1 \mathrm{~nm}$. Further increase in zein concentration to $30 \mathrm{mg} / \mathrm{mL}$ did not result in any change in particle size. Increasing zein concentration resulted in more zein and artemether molecules coming together to form larger particles. This is because in order to accommodate the increasing amount of zein and artemether in the colloidal system, larger particles were formed to reduce the overall area to volume ratio of the nanosuspension [20]. Increasing zein concentration also led to an increase in the viscosity of the nanosuspension, resulting in the formation of larger particles during nucleation. Similar observations have been reported before [20,21]. The PDI was observed to be below $0.14 \pm 0.03$.

\subsubsection{Ethanol Concentration}

The impacts of ethanol concentration on the size and PDI of artemether-loaded zein nanoparticles are presented in Figure 4d. Increasing the ethanol content from 50 to $90 \% \mathrm{v} / \mathrm{v}$ led to a decrease in particle size of the nanoparticles from about $163 \pm 16$ to $140 \pm 1 \mathrm{~nm}$. PDI also showed a similar pattern with the smallest particle observed at $90 \%$. The higher the solubility of zein in an ethanol-water binary solvent system, the smaller the particle size of nanoparticles produced [22]. The results indicated that zein was most soluble with a $90 \%$ ethanol content. Since there was no significant difference between the size of nanoparticles obtained at 80 and $90 \%$ ethanol contents, the former was selected for next optimization steps.

In summary, higher sodium caseinate/zein mass ratio, higher zein/artemether mass ratio, lower zein concentration and higher ethanol content favored the formation of smaller nanoparticles.

\subsection{Drug Content and Encapsulation Efficiency}

To evaluate the drug loading capacity and encapsulation efficiency, the artemetherloaded zein nanoparticles were prepared using a sodium caseinate/zein mass ratio of 1:1, a zein concentration of $7.5 \mathrm{mg} / \mathrm{mL}$, an ethanol concentration of $80 \%(v / v)$ and zein/artemether ratios varying from 2.5:1 to 20:1. The results are listed in Table 1.

Table 1. Artemether content and Encapsulation Efficiency (EE) *.

\begin{tabular}{cccc}
\hline $\begin{array}{c}\text { Zein/Artemether } \\
\text { Ratio }\end{array}$ & $\begin{array}{c}\text { Artemether Content } \\
(\mathbf{\%} w / w)\end{array}$ & $\begin{array}{c}\text { Encapsulated Drug } \\
\mathbf{( \% ~} \boldsymbol{w} / \boldsymbol{w})\end{array}$ & $\begin{array}{c}\text { EE } \\
\mathbf{( \% )}\end{array}$ \\
\hline $2.5: 1$ & $16.3 \pm 0.7$ & $5.07 \pm 0.32$ & $31.0 \pm 2.4$ \\
\hline $3.3: 1$ & $12.8 \pm 0.4$ & $5.86 \pm 0.31$ & $45.9 \pm 1.9$ \\
\hline $5: 1$ & $8.90 \pm 0.20$ & $4.64 \pm 0.17$ & $52.1 \pm 2.0$ \\
\hline $10: 1$ & $4.60 \pm 0.20$ & $2.53 \pm 0.17$ & $56.6 \pm 2.5$ \\
\hline $20: 1$ & $2.42 \pm 0.09$ & $1.51 \pm 0.07$ & $62.3 \pm 3.0$ \\
\hline
\end{tabular}

${ }^{*} \mathrm{EE}$ is the ratio between the amount of encapsulated artemether and the total amount of artemether in nanoparticles; Data are presented as mean $\pm \mathrm{SD}(n=3)$. 


\subsection{Physical Characterization}

The following preparation parameters, i.e., a sodium caseinate/zein mass ratio of 1:1, a zein/artemether mass ratio of 5:1, a zein concentration of $7.5 \mathrm{mg} / \mathrm{mL}$ and an ethanol concentration of $80 \%(v / v)$, were chosen to prepare artemether-load zein nanoparticles for further physical characterization using in vitro and in vivo testing.

\subsubsection{Particle Morphology}

The morphology of the typical artemether-load zein nanoparticles powder was examined and is presented in Figure 6. The nanoparticles appeared leafy at lower magnifications $(250 \times$ and $1000 \times)$. At higher magnifications, the nanoparticles were observed to be spherical with rough surfaces. Particle size was estimated to be around $150 \mathrm{~nm}$ confirming particle size analyses by dynamic light scattering. Nanoparticles prepared with different formulation parameters reported in this work exhibited similar morphologies (images not shown).

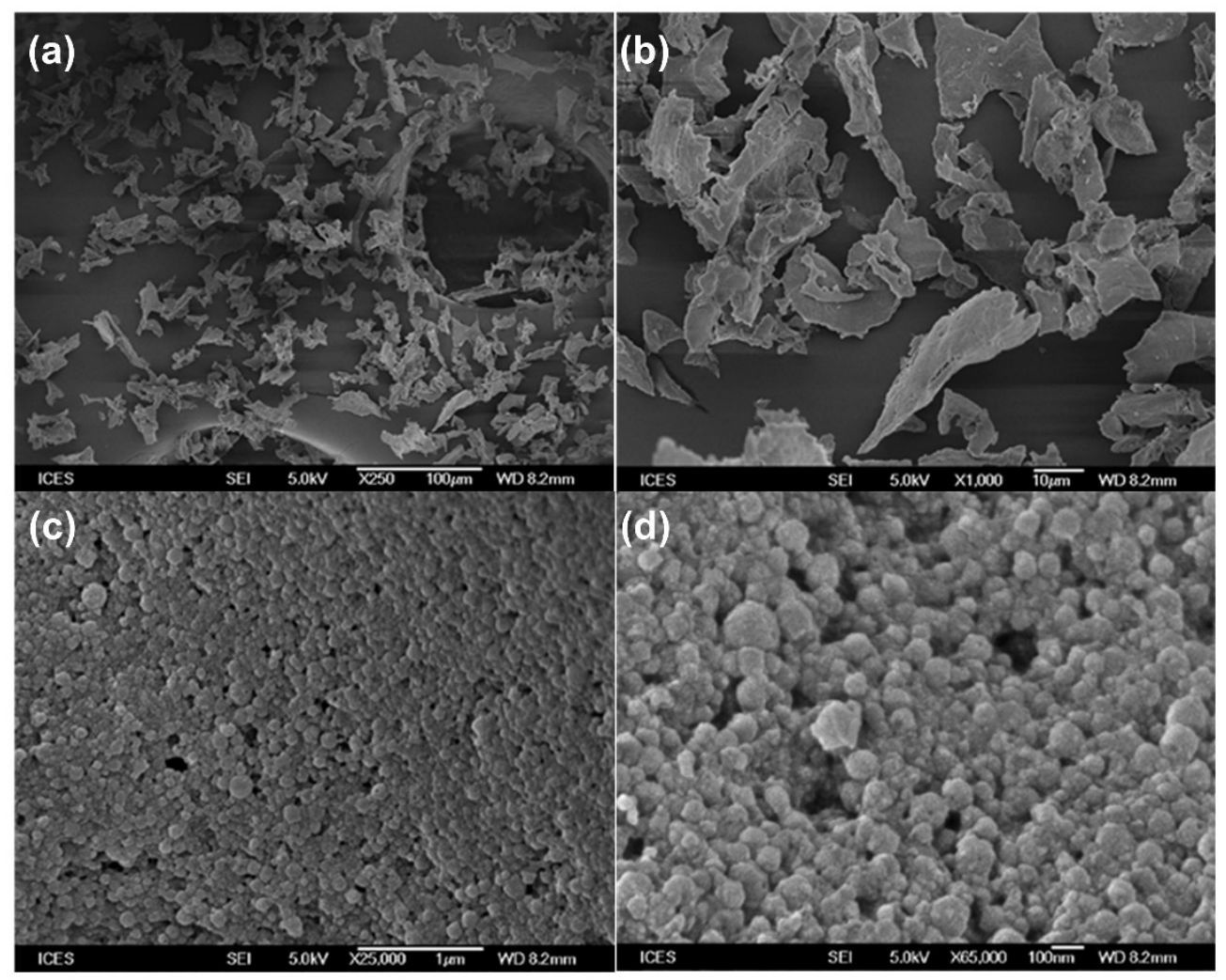

Figure 6. Scanning electron microscopic images of artemether-loaded zein nanoparticles powder at different magnifications. Image (a): 250×; (b): 1000×; (c) 25,000×; (d): 65,000×.

\subsubsection{Thermal Analysis}

The thermograms of raw artemether, artemether-loaded zein nanoparticles, sodium caseinate, zein and their physical mixture (mass ratio of 1:5:5) are displayed in Figure 7a. Zein and sodium caseinate did not exhibit any observable thermal peaks, implying that no physical transition occurred in the investigated range. Raw artemether exhibited the characteristic melting peak at $88^{\circ} \mathrm{C}$ with an enthalpy of $104 \mathrm{~J} \cdot \mathrm{g}^{-1}$. In the thermogram of the physical mixture, the endothermic peak of artemether was broad and shifted to $86^{\circ} \mathrm{C}$ with an enthalpy of $83 \mathrm{~J} \cdot \mathrm{g}^{-1}$. This shift and broadening of the endothermic peak together with the slight reduction in enthalpy observed were probably due to the presence of zein and sodium caseinate, which served as impurities. The melting peak in the thermogram of artemether-loaded zein nanoparticles was broadened and shifted to $85^{\circ} \mathrm{C}$. In addition, its enthalpy was decreased to $25 \mathrm{~J} \cdot \mathrm{g}^{-1}$, indicating significant amorphization of artemether. 
(a)

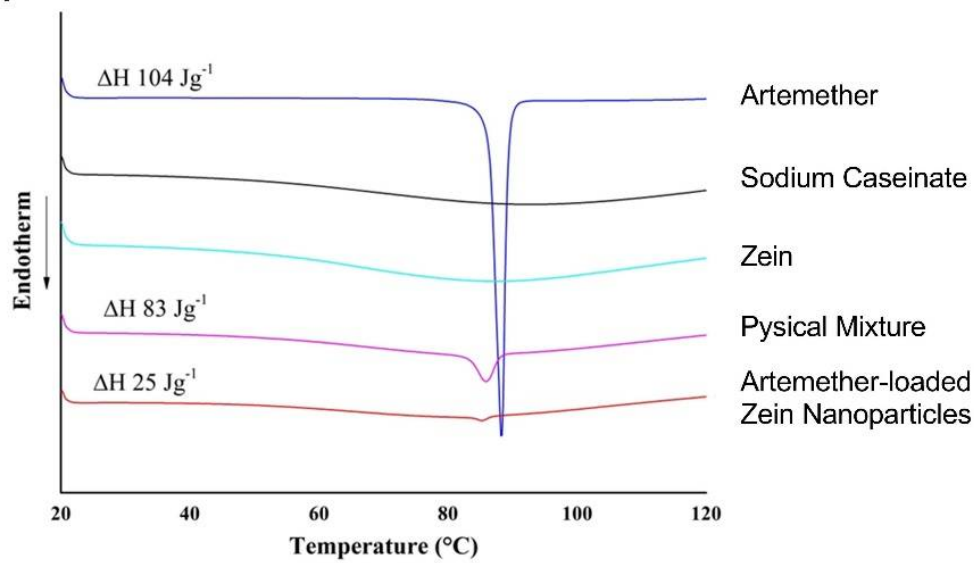

(b)

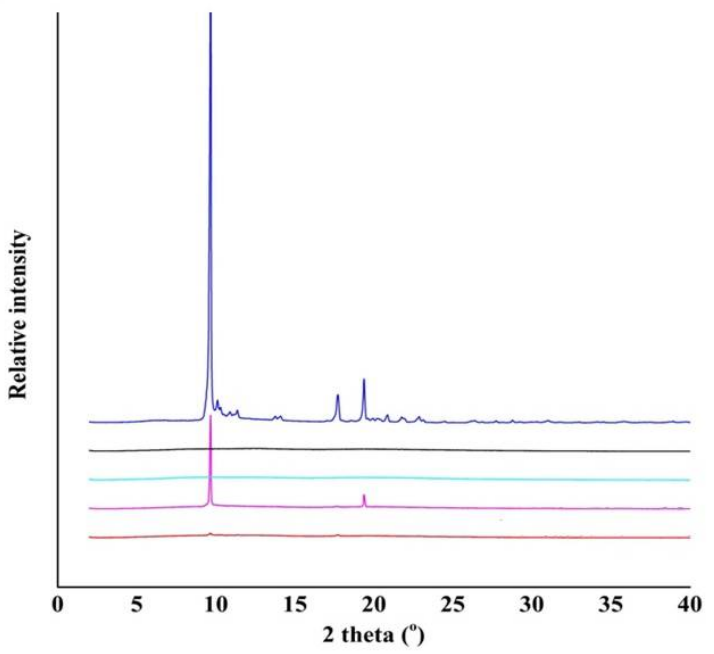

Artemether

Sodium Caseinate

Zein

Pysical Mixture

Artemether-loaded

Zein Nanoparticles

(c)

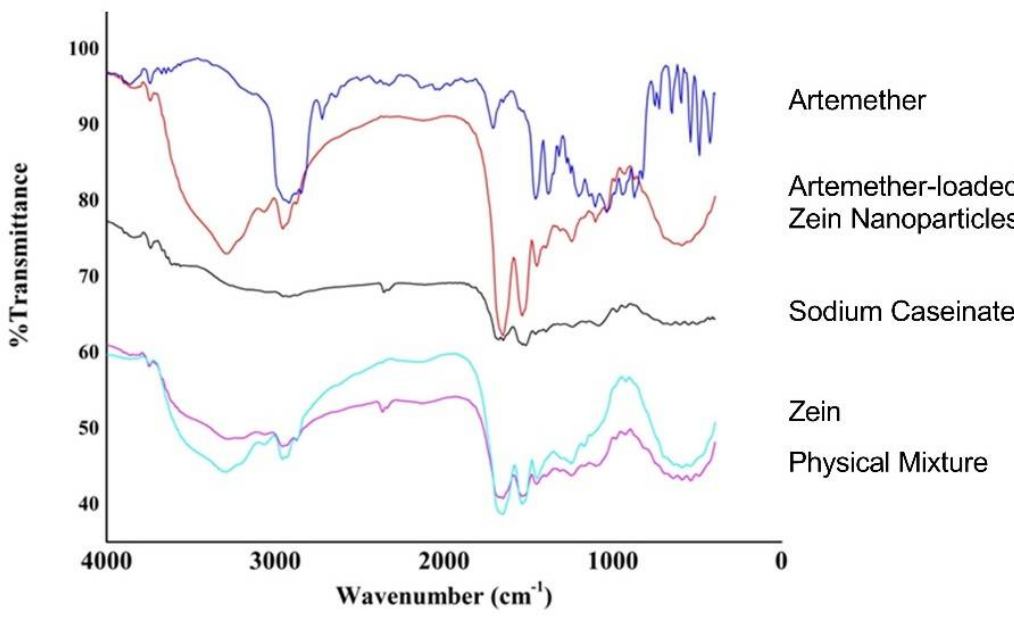

Figure 7. Physical characterization of artemether-loaded zein nanoparticles. Image (a) thermograms; (b) X-ray powder diffractograms; (c) Fourier transform infrared spectra of raw artemether, artemetherloaded zein nanoparticles, sodium caseinate, zein and their physical mixture (mass ratio of 1:5:5).

\subsubsection{Crystallinity}

The X-ray powder diffractograms of raw artemether, artemether-loaded zein nanoparticles, sodium caseinate, zein and their physical mixture (mass ratio of 1:5:5) are displayed in Figure $7 \mathrm{~b}$. Zein and sodium caseinate did not show any distinct peaks due to their 
amorphous natures. Raw artemether exhibited two distinct peaks at $2 \theta$ values of $9.66^{\circ}$ and $19.38^{\circ}$, which are characteristic of crystalline artemether. These peaks were also observed in the physical mixture. No characteristic artemether peak was observed in the artemether-loaded zein nanoparticles, suggesting extensive amorphization of artemether in the nanoparticles. This agrees with the results observed in the thermal analysis.

\subsubsection{Fourier Transform Infrared (FTIR) Spectra}

The FTIR spectra of raw artemether, artemether-loaded zein nanoparticles, sodium caseinate, zein and their physical mixture (mass ratio of 1:5:5) are shown in Figure 7c. Characteristic artemether bands were recorded at 2937, 1455 and $1036 \mathrm{~cm}^{-1}$, representative of $\mathrm{C}-\mathrm{H}$ stretching, $\mathrm{C}-\mathrm{H}$ bending and $\mathrm{C}-\mathrm{O}-\mathrm{C}$ stretching vibrations, respectively [23]. The FTIR spectra of artemether-loaded zein nanoparticles showed peaks at 2947 (C-H stretching vibration), 1450 (C-H bending vibration) and $1034 \mathrm{~cm}^{-1}$ (C-O-C stretching vibration). The presence of these characteristic artemether bands in the artemether-loaded zein nanoparticles suggests that artemether remained intact in the formulation and underwent insignificant degradation.

\subsection{Release Profile}

The release profile of artemether-loaded zein nanoparticles is presented in Figure 8. Artemether was found to be released from the nanoparticles through a biphasic pattern with an initial burst release phase, followed by a slow-release phase. Artemether of $47 \pm$ $1 \%$ was released in the first $15 \mathrm{~min}$. The release was gradually increased to $99 \pm 2 \%$ in 24 $\mathrm{h}$. The initial rapid release represented the burst release of unencapsulated artemether in the formulation. Encapsulated artemether was released slowly, accounting for the second phase in the release profile since drug molecules were embedded in a matrix with zein in the nanoparticle structure. The extended-release property of the nanoparticles could be attributed to the hydrophobic nature of zein, which slowed down the penetration of water into the nanoparticles. Unlike another study [24], the unencapsulated drug was allowed to remain in the formulation. This is because a high initial burst release is beneficial in terms of achieving a therapeutic concentration of artemether in the bloodstream immediately after administration. Probably due to inferior aqueous solubility, raw artemether exhibited very slow dissolution in the first $2 \mathrm{~h}$. However, almost all raw artemether was dissolved within $5 \mathrm{~h}$ while only $54 \pm 2 \%$ of artemether was released from the nanoparticles during the same period. The biphasic and extended-release behaviour of artemether-loaded zein nanoparticles may lead to better therapeutic efficacy in clinical settings for severe malaria patients under emergency.

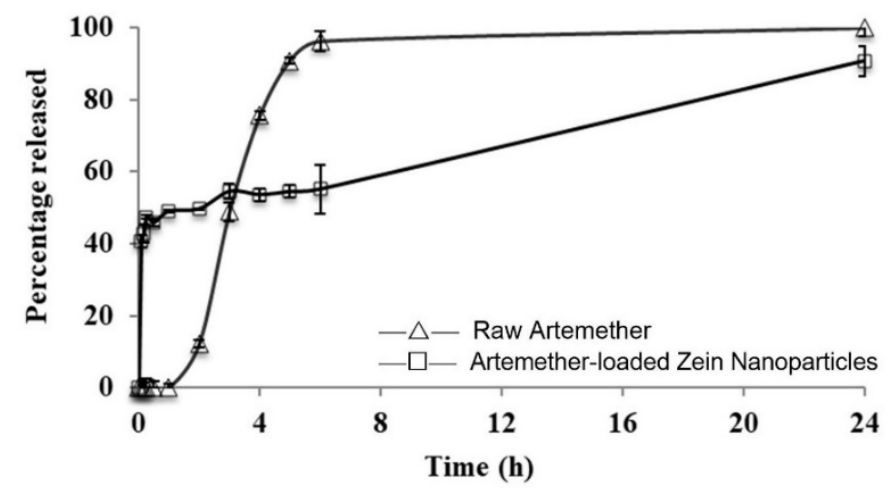

Figure 8. Release profiles of artemether from artemether-loaded zein nanoparticles and raw artemether in phosphate buffer solution (PBS) at $37^{\circ} \mathrm{C}$. All experiments were carried out in triplicate $(n=3)$; symbols represent mean values while error bars represent $\mathrm{SD}$. 


\subsection{Physical Stability}

Physical stability of lyophilized artemether-loaded zein nanoparticles powder on storage and after reconstitution with either Milli-Q water or normal saline was examined. The study was performed by assessing change in particles size, zeta potential and encapsulation efficiency. All zeta potential measurements were taken at $\mathrm{pH} 6.3$ and 5.9 for powder reconstituted with Milli-Q water and normal saline, respectively. Electrostatic repulsion between nanoparticles measured as zeta potential stabilizes them by preventing aggregation and particle growth. Stable colloidal systems possess zeta potential values above $-25 \mathrm{mV}$ [25] When artemether-loaded zein nanoparticles were stored at $4{ }^{\circ} \mathrm{C}$ and $75 \%$ relative humidity for 24 weeks, the encapsulation efficiency remained unchanged; the particle size remained below $160 \mathrm{~nm}$, while the zeta potential fell in the range of $-40 \pm 2 \mathrm{mV}$ throughout the storage duration (Figure 9a), suggesting their excellent physical stability.

(a)

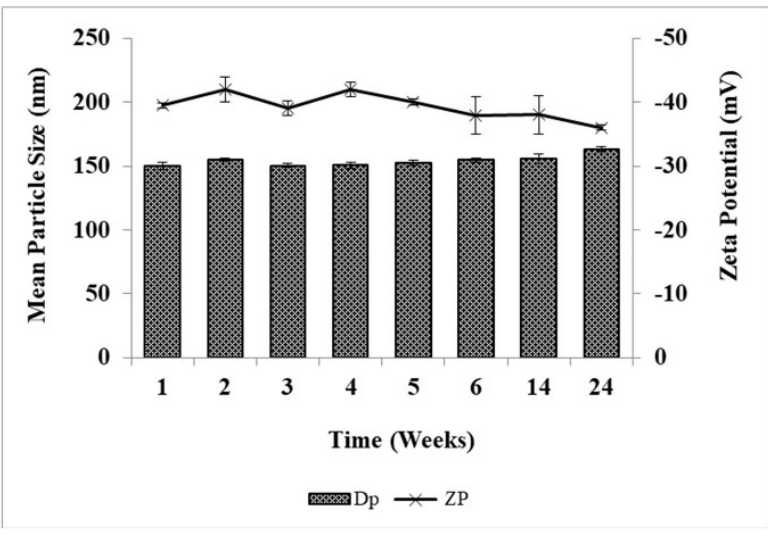

(b)

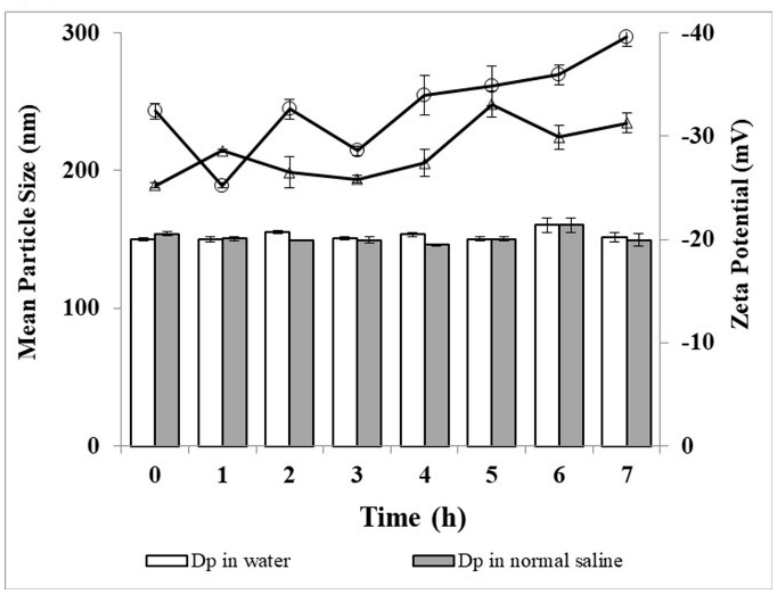

Figure 9. Physical stability of artemether-loaded zein nanoparticles. (a) Particle size (DP) and zeta potential (ZP) analyses of artemether-loaded zein nanoparticles powder on storage at $4{ }^{\circ} \mathrm{C}$ and relative humidity of $75 \pm 5 \%$ for 24 weeks; (b) particle size and zeta potential analyses of artemetherloaded zein nanoparticles after reconstitution with water and with normal saline. All experiments were carried out in triplicate $(n=3)$; symbols represent mean values while error bars represent SD.

Artemether-loaded zein nanoparticles also showed a high level of stability after reconstitution with Milli-Q water or normal saline as no significant increase in particle size was found (Figure $9 \mathrm{~b}$ ). The size of artemether-loaded zein nanoparticles remained below $160 \mathrm{~nm}$ for $7 \mathrm{~h}$ after reconstitution with Milli-q water or normal saline. Zeta potential values of artemether-loaded zein nanoparticles reconstituted with normal saline were generally lower than that of Milli-Q water. Normal saline has a high ionic strength $(154 \mathrm{mEq} / \mathrm{L}$ of 
$\mathrm{Na}^{+}$and $\mathrm{Cl}^{-}$) that exerted shielding effect on nanoparticles after reconstitution, hence weakening the interparticular electrostatic repulsive forces. This could explain for the lower zeta potential values of normal saline reconstituted products [26].

\subsection{In Vitro Hemolysis Test}

As hemolysis is a common clinical phenomenon occurring after artemisinin-based therapy [11], it is of great interest to examine the hemolytic property of the artemether-loaded zein nanoparticles. An in vitro hemolysis test is commonly used to study the toxicity of a formulation on red blood cells (RBCs). It can also be used to estimate potential membrane damage caused by a parenteral dosage form [27]. The breakdown of RBCs is measured by the amount of haemoglobin released. Hemolytic tendencies of artemether-loaded zein nanoparticles (artemether: $2 \mathrm{mg} / \mathrm{mL}), 5 \%$ DMSO, sodium caseinate $(7.5 \mathrm{mg} / \mathrm{mL})$, sodium caseinate together with artemether $(2 \mathrm{mg} / \mathrm{mL})$ and artemether $(2 \mathrm{mg} / \mathrm{mL})$ in $5 \%$ DMSO were investigated. Phosphate buffered saline (PBS) and Triton X 100 were used as the negative and positive controls, respectively, as (1) PBS is assumed to cause no haemolysis and (2) Triton X 100 is a surfactant known to cause $100 \%$ hemolysis. Since artemether is insoluble in water, artemether was dissolved in 5\% DMSO.

Consistent with the clinical observations [11], artemether cased substantial hemolysis (Figure 10). Although 5\% DMSO had a mild hemolytic effect $(8.0 \pm 0.6 \%)$, when artemether was solubilized in $5 \%$ DMSO, it destroyed RBCs as much as the positive control. Of note, sodium caseinate also caused significant hemolysis $(32.0 \pm 6.2 \%)$. However, when the hemolytic artemether and sodium caseinate were formulated into nanoparticles with zein, the hemolytic effect dropped by at least 15 -fold $(6.0 \pm 1.0 \%)$. Obviously, a less hemolytic intravenous dosage of artemether was enabled by the nanoparticles.

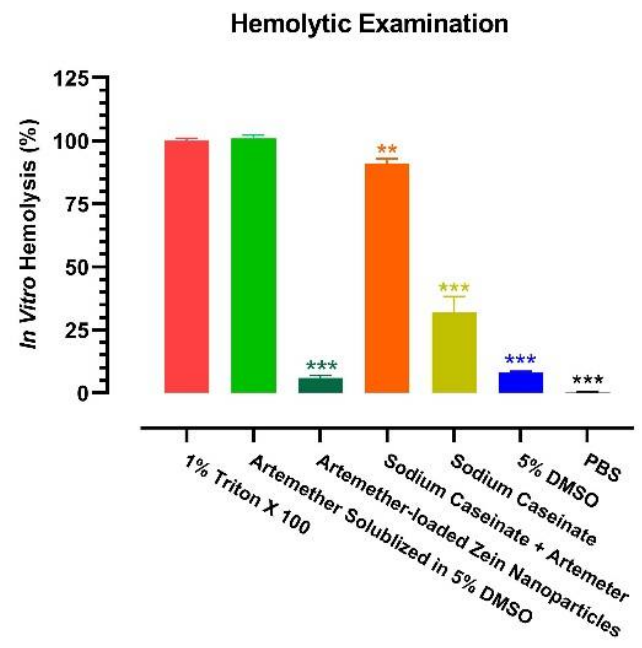

Figure 10. Hemolytic property of artemether-loaded zein nanoparticles. The hemolytic tendency was examined by in vitro hemolysis test. All experiments were carried out in triplicate $(n=3)$; symbols represent mean values while error bars represent SD. ${ }^{* *} p<0.01$, ${ }^{* * *} p<0.001$ between this group and $1 \%$ Triton X 100, two-tailed unpaired $t$-test.

\subsection{Pharmacokinetic Study}

The pharmacokinetic profiles of artemether were assessed after single bolus intravenous administration at the dose of $5 \mathrm{mg} / \mathrm{kg}$ using two different formulations-namely, free artemether solution formulated with cosolvency and artemether-loaded zein nanoparticles. The plasma artemether concentration-time data are shown in Figure 11, while the major intravenous pharmacokinetic parameters are listed in Table 2. 


\section{Intravenous Pharmacokinetic Profile}



Figure 11. Intravenous pharmacokinetic profiles of artemether. The male Sprague-Dawley rats received respective intravenous administration of artemether in nanoparticles or cosolvent formulation at $5 \mathrm{mg} / \mathrm{kg}$. Data are presented as mean $\pm \mathrm{SD} ; n=5$ except $n=3$ at $420 \mathrm{~min}$ for rats that received artemether in cosolvent. ${ }^{*} p<0.05$ between nanoparticle group and cosolvent group, two-tailed unpaired $t$-test.

Table 2. Intravenous pharmacokinetic parameters of artemether ${ }^{\mathrm{a}}$.

\begin{tabular}{ccc}
\hline Formulation & $\begin{array}{c}\text { Artemether Solubilized in } \\
\text { Cosolvent }\end{array}$ & $\begin{array}{c}\text { Artemether-Loaded Zein } \\
\text { Nanoparticles }\end{array}$ \\
\hline Dose $(\mathrm{mg} / \mathrm{kg})$ & 5 & 5 \\
\hline$V_{c}(\mathrm{~L} / \mathrm{kg})$ & $2.14 \pm 0.98$ & $1.95 \pm 0.85$ \\
\hline$A U C_{0 \rightarrow \text { last }}\left(10^{4} \times \mathrm{min} \cdot \mathrm{ng} / \mathrm{mL}\right)$ & $6.22 \pm 1.22$ & $8.14 \pm 4.52$ \\
\hline$C L(\mathrm{~mL} / \mathrm{min} / \mathrm{kg})$ & $83.1 \pm 17.4$ & $72.4 \pm 25.7$ \\
\hline$t_{1 / 2 \lambda Z}(\mathrm{~min})$ & $165 \pm 47$ & $297 \pm 132$ \\
\hline$M R T_{0 \rightarrow \text { last }}(\mathrm{min})$ & $45.6 \pm 16.4$ & $82.9 \pm 15.2 * *$ \\
\hline a
\end{tabular}

a Results are presented as Mean \pm
artemether solubilized in cosolvent.

Similar to the results observed in a previous study [28], artemether was eliminated from plasma through a biexponential process-i.e., a rapid distribution phase followed by a prolonged terminal elimination phase. Therefore, the plasma artemether concentration-time data of the respective rat were fitted into the classical two-compartment first-order open model $[29,30]$. When it was given in free solution form, artemether was found to have a moderate apparent volume of distribution of the central compartment $\left(V_{c}=2.14 \pm 0.98 \mathrm{~L} / \mathrm{kg}\right)$, a very rapid clearance $(C L=83.1 \pm 17.4 \mathrm{~mL} / \mathrm{min} / \mathrm{kg})$ and a moderate terminal elimination half-life $\left(t_{1 / 2 \lambda Z}=167 \pm 47 \mathrm{~min}\right)$ and mean residence time $(M R T=45.6 \pm 16.4 \mathrm{~min})$. When nanoparticles were given intravenously, although the $V_{c}$ value was similar $(1.95 \pm 0.85$ vs. $2.14 \pm 0.98 \mathrm{~L} / \mathrm{kg}$ ), artemether tended to display a longer $t_{1 / 2} \lambda Z$, slower $C L$ but more abundant plasma exposure. Of note, the nanoparticles led to $~ 80 \%$ increase in the MRT of artemether ( $82.9 \pm 15.2$ vs. $45.6 \pm 13.4 \mathrm{~min}$, two-tailed independent $t$-test: $p=0.0057)$. Moreover, at 300 and $420 \mathrm{~min}$ after administration, plasma artemether levels in rats that received nanoparticles were significantly higher than that in rats that received artemether solution $(p<0.05)$. Similarly, at $600 \mathrm{~min}$ after intravenous administration, artemether remained measurable in all rats received nanoparticles $(13.8 \pm 4.1 \mathrm{ng} / \mathrm{mL})$; however, plasma level of artemether dropped to unmeasurable levels $(<10 \mathrm{ng} / \mathrm{mL})$ in all rats that received free artemether solution. Clearly, the extended-release effect of artemether-loaded zein nanoparticles was confirmed by in vivo study. 


\section{Discussion}

According to the treatment guidelines issued by the WHO [7], parenteral artemisinin derivatives were used to manage severe malaria. Intravenous/intramuscular administration of water-soluble artesunate is the first choice. When parenteral artesunate is not available, an oil-based intramuscular injection of artemether can be used. Besides injection pain, the oil-based dosage form of artemether is associated with slow absorption and erratic pharmacokinetics, which adversely affect its clinical efficacy. In the present study, we formulated artemether into an intravenous dosage form using nanotechnology. For the management of life-threatening severe malaria, an intravenous formulation is obviously superior to an intramuscular dosage form as it leads to immediate onset antimalarial effects with higher and more consistent blood exposure, consequently decreasing the mortality. Our findings shed light on an innovative therapeutic option for severe malaria.

It is well known that artemisinin and its derivatives, including artesunate, artemether and dihydroartemisinin, are short-acting antimalarial agents that kill parasites more rapidly than other antimalarials, and are active against both the asexual and sexual stages of the parasite life-cycle [31]. The metabolism of artesunate and artemether have been well elucidated and both of them can be considered as a prodrug of dihydroartemisinin because they are rapidly hydrolyzed to dihydroartemisinin upon dosing [31]. It has been reported that artemether displayed a longer half-life than regardless of whether intravenous administration or oral dosing was used $[28,32,33]$. Since dihydroartemisinin, the active metabolite of artesunate/artemether, is also short-acting, a parent drug with a longer half-life is preferable as it will lead to a blood artemisinin derivative(s) level above the minimal effective concentration for a longer duration and reduced dosing frequency, consequently enhancing the therapeutic efficacy. From this angle, artemether appears to be a therapeutic entity superior to artesunate. However, in current clinical practice, intravenous artesunate, which commonly displays a half-life less than $1 \mathrm{~h}$ [33], is the first choice for severe malaria [7]. This is probably due to the solubility issue of artemether, which has hindered its intravenous administration. The artemether-loaded zein nanoparticles developed in the present study may enable intravenous delivery and change its role in the management of serve malaria.

The applications of nanoparticles to deliver artemether have been extensively attempted using different excipients and preparation methods and promising results were commonly reported [10,34-42]. However, most of the previous studies were focused on oral, transdermal or other nonparenteral delivery. So far, the intravenous dosage forms of artemether-loaded nanoparticles have only been attempted in a few studies using human serum albumin or glycerol trimyristate/monostearate plus soybean oil as carriers $[10,39,40,43]$. Again, such injectable formulations exhibited superior antimalarial efficacies and/or reduced toxicities in previous studies. However, as malaria is a disease of poverty $[4,5]$, excipients with higher affordability such as zein and sodium caseinate are more cost-effective. To the best of the authors' knowledge, this is the first attempt to use zein and sodium caseinate as major excipients to formulate artemether into a nanoformulation.

In the present study, the zein nanoparticles were found to release artemether through a biphasic process-i.e., a burst release phase followed by a slow-release phase. The burst release of artemether was probably due to the dissolution of unencapsulated artemether attached to the surface of the nanoparticles in PBS/bloodstream while the slow release was probably attributed to the release of artemether embedded in the core of the nanoparticles. As zein is hydrophobic, the penetration of water into the nanoparticles appears to be a slow process, resulting in the extended-release of artemether. As an intravenous dosage form, such biphasic release profile was favorable as it enabled immediate therapeutic effects upon administration and prolonged the therapeutic period.

Our explanations on the release profile were well supported by the pharmacokinetic data. When compared to the artemether delivered by cosolvent, artemether-loaded zein nanoparticles did not lead to an alternation in the $V_{c}$. This phenomenon could be well explained by the burst release of artemether. As we knew, $\sim 50 \%$ of artemether associated with the nanoparticles was not encapsulated, so upon intravenous administration, this portion 
of artemether would leave the nanoparticles and enter the bloodstream rapidly, resulting in a similar pharmacokinetic profile in the first hour after administration. In comparison to the artemether delivered by cosolvent, the formulation of nanoparticles substantially slowed down the decline of the plasma artemether level at terminal elimination stage and dramatically increased the MRT. The prolongation of residence of artemether could be explained by the extended-release of artemether from the nanoparticles, leading to a longer circulating period in bloodstream. An intravenous dosage form of artemisinin derivative with extended-release property is highly favorable as all these therapeutic agents suffer from rapid clearance and short half-lives, and prolonged infusion is required if the drug is given through an intravenous route. Hopefully, the artemether-loaded zein nanoparticles may decrease the dosing frequency and offer a more patient-friendly therapeutic option.

During the past two decades, nanomedicines have been extensively attempted in cancer therapy. Such approaches may increase drug accumulation through enhanced permeability and retention in tumors to improve anticancer efficacy and provide long systemic circulation of entrapped drug with high plasma concentration [44]. Similarly, our zein nanoparticles substantially prolonged the systemic circulation of artemether, an antimalarial agent with a very short half-life. However, the impact of zein nanoparticles on artemether accumulation in its target sites remains unclear. As artemisinin derivatives can kill the parasites at both human liver and blood stages [31], liver and red blood cells can be considered as their targets organ/cells. Although we attempted to measure artemether and its active metabolite dihydroartemisinin in red blood cells and plasma when we developed the assay, we only managed to quantify artemether in plasma. Therefore, whether artemether-loaded zein nanoparticles enhanced artemether accumulation remains unclear. Of note, in a previous study, human serum albumin nanoparticles substantially increased the penetration of artemether to RBCs and enhanced its antimalarial efficacy in mice [40]. Similarly, as zein nanoparticles enhanced liver uptake in various previous studies [24,45-47], it appears to be a practical strategy for hepatic targeting, which is highly favorable for the treatment of malaria.

In clinical management of malaria, hemolysis commonly occurred after artemisininbased therapy [11]. A recent study suggested that the hemolysis was malaria-independent and mediated through a toxic oxidative effect of artemisinin derivative(s) on the red blood cell membrane as malaria-free rats receiving artesunate also suffered from hemolysis [48]. In the present study, we confirmed that free artemether was highly hemolytic in our in vitro test. Similarly, the excipient sodium caseinate also caused hemolysis. Interestingly, when artemether and sodium caseinate were formulated into zein nanoparticles, their hemolytic effects were substantially masked. Probably through the formation of nanoparticles, sodium caseinate and $\sim$ half of the artemether were sealed inside the nanoparticles, minimizing the amounts of free sodium caseinate and artemether. Moreover, in our pharmacokinetic study, hemolysis was not observed in blood samples collected from rats receiving artemether-loaded zein nanoparticles but observed in some rats receiving artemether solubilized in cosolvent (data not shown). Clearly, artemether-loaded zein nanoparticles were less hemolytic. However, the beneficial effect of artemether-loaded zein nanoparticles on hemolysis needs to be confirmed in a study where the animals receive repeated dosing as artemisinin associated hemolysis commonly had a delayed onset after repeated dosing in clinical settings [11].

Due to the constrained resources, the antimalarial effects of artemether-loaded zein nanoparticles were not attempted in the present study. It would be of great scientific interest to assess its parasite-killing activities in cell culture models and examine therapeutic efficacy in malaria-bearing rodents. Similarly, the preclinical safety/toxicological profile should be carefully examined.

In summary, an innovative intravenous dosage form of artemether was formulated using artemether-loaded zein nanoparticles. This formulation was found to display good encapsulation efficiency, excellent physical stability and offer extended-release property. Encapsulation of artemether into zein nanoparticles strongly suppressed in vitro hemolysis. 
Upon intravenous administration, artemether-loaded zein nanoparticles substantially prolonged the circulation of artemether in the bloodstream, suggesting that the nanoparticles may enhance its therapeutic efficacy in clinical settings. In conclusion, intravenous delivery of artemether-loaded zein nanoparticles appears to be a promising therapeutic option for the management of severe malaria.

\section{Materials and Methods}

\subsection{Materials}

Artemether was supplied by Biotain Pharma Co., Ltd. (Xiamen, China). Zein was a kind gift from Flo Chemical Corporation (Ashburnham, MA, USA). Sodium caseinate was purchased from Sigma-Aldrich (St. Louis, MO, USA). Absolute ethanol was obtained from VWR Singapore Ltd. (Singapore). Ultrapure water was prepared by a Elix ${ }^{\circledR}$ Essential 5 UV Water Purification System (Molsheim, France) or a Millipore Direct- $Q^{\circledR}$ Ultra-Pure Water System (Billerica, MA, USA) and used in all experiments. High performance liquid chromatography (HPLC) grade acetonitrile was supplied by Tedia (Fairfield, OH, USA). All other chemicals are of reagent grade and were obtained from either Sigma-Aldrich or Tokyo Chemical Industry (Tokyo, Japan).

\subsection{Preparation of Artemether-Loaded Zein Nanoparticles}

Artemether-loaded zein nanoparticles were prepared by a modified antisolvent precipitation approach [14]. Briefly, zein (50-300 mg) and artemether (0-30 mg) were dissolved in $5 \mathrm{~mL}$ of the ethanol-water binary solvent system containing $50,60,70,80$, and $90 \% v / v$ ethanol. Sodium caseinate $(0-150 \mathrm{mg})$ was dissolved in $10 \mathrm{~mL}$ water. In total, $10 \mathrm{~mL}$ of aqueous sodium caseinate solution was added instantly to $5 \mathrm{~mL}$ zein solution with a $1000 \mathrm{rpm}$ magnetic stirring. Upon mixing, artemether-loaded zein nanoparticles precipitated immediately. Ethanol was then removed from the nanosuspension either by normal evaporation with magnetic stirring or rotary evaporation (Rotavapor ${ }^{\circledR}$ R-205, BUCHI Labortechnik AG, Flawil, Switzerland) for $1-5 \mathrm{~h}$. The prepared nanosuspension was frozen in liquid nitrogen and lyophilized for $48 \mathrm{~h}$. The achieved artemether-loaded zein nanoparticles powders were stored at $4{ }^{\circ} \mathrm{C}$ until further analysis.

\subsection{Particle Size and Zeta Potential Analyses}

Particle size of artemether-loaded zein nanoparticles was analyzed by dynamic light scattering (DLS) using Zetasizer Nano ZS (Malvern, UK) [10]. In brief, nanoparticles were dispersed in water to make the equivalent of $0.2 \mathrm{mg}$ zein $/ \mathrm{mL}$. Scattering angle and temperature were set to $175^{\circ}$ and $25^{\circ} \mathrm{C}$, respectively. Each sample was equilibrated for $120 \mathrm{~s}$ and data were collected over 5 sequential readings. Measurements were performed in triplicate. Mean particle size (Z-average, diameter) and standard deviation were calculated. Zeta potential was measured by electrophoretic light scattering technology using Zetasizer Nano ZS (Malvern, UK). Nanoparticles were diluted to the equivalent of $0.2 \mathrm{mg}$ zein $/ \mathrm{mL}$, as was carried out for particle size analysis. Measurements were carried out in triplicate and the mean and standard deviation were calculated.

\subsection{Drug Content and Encapsulation Efficiency}

To obtain the total artemether content in the lyophilized artemether-loaded zein nanoparticles powder, $90 \% v / v$ ethanol-water binary solvent system was used to reconstitute the powder. After sonication for $30 \mathrm{~min}$, the suspension was centrifuged at 10,800 rpm for $30 \mathrm{~min}$. The supernatant was collected and assayed using high performance liquid chromatography (HPLC) analysis. A Zorbax Eclipse C18 reversed phase column $(4.6 \times 50 \mathrm{~mm}$, $5 \mu \mathrm{m}$, Agilent, USA) was used with 70\% acetonitrile plus $30 \% 0.01 \mathrm{M} \mathrm{KH}_{2} \mathrm{PO}_{4}$ solution $(\mathrm{pH} 4)$ as the mobile phase. Drug content was calculated using the formula below:

$$
\text { Drug Content }(\%)=\frac{\text { Amount of artemether in nanoparticles }}{\text { The amount of nanoparticles }} \times 100 \%
$$


To evaluate the encapsulation efficiency, ethyl acetate was used to extract unencapsulated artemether from the artemether-loaded zein nanoparticles powder [10]. Ethyl acetate was the solvent of choice since artemether is soluble in it but zein is not [14]. Accurately weighed artemether-loaded zein nanoparticle powder was dispersed in $1 \mathrm{~mL}$ ethyl acetate for $1 \mathrm{~min}$. The supernatant was collected and analyzed in the same way as the drug content. The amount of artemether encapsulated was calculated by deducting the amount of unencapsulated artemether from the drug content in the artemether-loaded zein nanoparticles. Encapsulation efficiency (EE) was calculated using the formula below:

$$
\text { Encapsulation efficiency }(\%)=\frac{\text { Amount of encapsulated artemether }}{\text { Total amount of artemether in zein nanoparticles }} \times 100 \%
$$

\subsection{Particle Morphology}

The morphology of artemether-loaded zein nanoparticles was observed using field emission scanning electron microscope (FESEM, JEOL JSM-6700F, Tokyo, Japan) at a $5 \mathrm{kV}$ acceleration voltage [10]. Prior to visualization, the sample was placed on double sided copper tape mounted on metal stubs. It was then sputtered with gold using Cressington 208HR (Ted Pella, Inc., UK) at $10 \mathrm{~mA}$ for $120 \mathrm{~s}$.

\subsection{Thermal Analysis}

Differential scanning calorimetry (DSC) was employed to study the thermal properties of artemether-loaded zein nanoparticles powder, raw artemether, zein, sodium caseinate and their physical mixture at the mass ratio 1:5:5 [10]. Diffractograms were taken using a Mettler Toledo DSC 1 (Mettler-Toledo AG, Analytical, Schwerzenbach, Switzerland). Samples of 2-5 mg were sealed in an aluminium crucible and heated at a rate of $10^{\circ} \mathrm{C} / \mathrm{min}$ over a temperature range of 30 to $120^{\circ} \mathrm{C}$ under $10 \mathrm{~mL} / \mathrm{min} \mathrm{N}_{2}$.

\subsection{Crystallinity}

Crystallinities of artemether-loaded zein nanoparticles powder, raw artemether, zein, sodium caseinate and their physical mixture at mass ratio 1:5:5 were investigated using a powder X-ray diffractometer (D8-Advance, Bruker AXS GmbH, Karlsruhe, Germany) with a PSD Vantec-1 detector [10]. Data were acquired over an angular range of $2^{\circ}-40^{\circ}(2 \theta)$ at a step of $0.017^{\circ}$ using monochromatized $\mathrm{CuK} \alpha$ radiation $(\lambda: 1.542 \AA)$ with $20 \mathrm{kV}$ and $40 \mathrm{~mA}$. Divergence and antiscattering slits were set at $0.3^{\circ}$.

\subsection{Drug-Protein Interaction}

Fourier transform infrared (FTIR) spectra of artemether-loaded zein nanoparticles powder, raw artemether, zein, sodium caseinate and their physical mixture at a mass ratio of 1:5:5 were acquired using an Excalibur FTS 3000 MX (Bio-Rad, Hercules, CA, USA) [10]. The sample to be investigated was mixed with $\mathrm{KBr}$ at a mass ratio 1:100 and compressed to form a disc prior to FTIR scanning. Pure KBr disc was used as the background. For each measurement, 64 scans were taken at a spectral resolution of $4 \mathrm{~cm}^{-1}$, over a wavenumber range of 400 to $4000 \mathrm{~cm}^{-1}$.

\subsection{In vitro Release Study}

The dissolution profile of artemether-loaded zein nanoparticles was studied by the "sample and separate" method [49]. In brief, artemether-loaded zein nanoparticle powders containing $6 \mathrm{mg}$ artemether were put in $100 \mathrm{~mL}$ phosphate buffered saline (PBS) of $\mathrm{pH} 7.4$. The study was performed under sink conditions at $37 \pm 0.5^{\circ} \mathrm{C}$ with $150 \mathrm{rpm}$ stirring. The beaker was covered with parafilm to minimize water loss via evaporation. In total, $1 \mathrm{~mL}$ samples were withdrawn at selected time points $(1 / 12,1 / 6,1 / 4,1 / 2,1,2,3,4,5,6,24 \mathrm{~h})$. Samples were centrifuged at 10,800 rpm for $30 \mathrm{~min}$ and the supernatants were analyzed by HPLC in the same way as drug content. Then, $1 \mathrm{~mL}$ PBS at $37^{\circ} \mathrm{C}$ was added after each sampling to maintain sink condition. The experiment was repeated 3 times to ensure 
consistency. Raw artemether was used as control. Mean values and standard deviations were subsequently calculated.

\subsection{In Vitro Hemolytic Test}

This test was performed to examine the hemolytic potential of artemether-loaded zein nanoparticles and the formulation ingredients on red blood cells (RBCs) [10]. Freshly collected rat blood was centrifuged at $1500 \times \mathrm{g}$ for $10 \mathrm{~min}$ at $4{ }^{\circ} \mathrm{C}$. The plasma was collected for other purpose. The remaining RBCs were washed with PBS and then centrifuged at $1500 \times g$ at $4{ }^{\circ} \mathrm{C}$ for $5 \mathrm{~min}$. After two repeats of such procedures, the RBCs were diluted 25 times with PBS to make a $4 \% v / v$ RBCs dispersion. Such RBCs were kept at $4{ }^{\circ} \mathrm{C}$ and used within $24 \mathrm{~h}$.

The impact of artemether-loaded zein nanoparticles containing $2 \mathrm{mg} / \mathrm{mL}$ artemether, raw artemether in 5\% $v / v$ aqueous DMSO solution $(2 \mathrm{mg} / \mathrm{mL}), 5 \% v / v$ aqueous DMSO solution, sodium caseinate $(7.5 \mathrm{mg} / \mathrm{mL})$ solution, artemether $(2 \mathrm{mg} / \mathrm{mL})$ together with sodium caseinate $(7.5 \mathrm{mg} / \mathrm{mL})$ solution was assessed.

PBS and $1 \% v / v$ Triton-X 100 solution were assessed by adding $1 \mathrm{~mL}$ of the test solutions each to $1 \mathrm{~mL}$ of the $4 \% v / v$ RBCs dispersion. Each sample was incubated with the $\mathrm{RBC}$ at $37^{\circ} \mathrm{C}$ for $1 \mathrm{~h}$ and then centrifuged at $1500 \times g$ for $10 \mathrm{~min}$. The absorbance of the supernatant was taken at $550 \mathrm{~nm}$ with U-2900 UV spectrophotometer (Hitachi, Japan). Percentage haemolysis was calculated as follows:



\subsection{Physical Stability}

Artemether-loaded zein nanoparticles were stored at $4{ }^{\circ} \mathrm{C}$ and $75 \%$ relative humidity and monitored for changes in particle size, zeta potential and encapsulation efficiency for 24 weeks. Samples were taken out at predetermined time points for particle size, zeta potential and encapsulation efficiency were examined. Stability of artemether-loaded zein nanoparticles after reconstitution with Milli-Q water or normal saline upon storage at $4{ }^{\circ} \mathrm{C}$ was studied by measuring particle size and zeta potential. Samples were taken at predetermined time points for particle size and zeta potential analyses over a $7 \mathrm{~h}$ period.

\subsection{Pharmacokinetic Study}

This pharmacokinetic study was conducted with strict adherence to the Guidelines on the Care and Use of Animals for Scientific Purposes (Singapore). The study design and animal handling procedures were reviewed and approved by the Institutional Animal Care and Use Committee of the National University of Singapore (NUS) (Project No.: R15-1273, 5 November 2015). All in vivo experiments were carried out in a specific pathogen-free animal facility (temperature: $22 \pm 1{ }^{\circ} \mathrm{C}$; humidity: 60-70\%) in Comparative Medicine, NUS [50,51]. Male Sprague-Dawley rats (9-10 weeks old, weight: 300-350 g) were ordered from InVivos (Singapore) through Comparative Medicine. The animals were housed under a $12 \mathrm{~h}$ light-dark cycle with free access to food and water. On the day before the pharmacokinetic study, surgery was performed and a catheter (polyethylene tube, i.d. $=0.580 \mathrm{~mm}$, o.d. $=0.965 \mathrm{~mm}$, Becton Dickinson, Sparks, MD, USA) was implanted into the right jugular vein under isoflurane anesthesia. Intravenous artemether administration and blood collection were carried out via this cannula. To prevent cross-contamination and blood clotting, $\sim 0.3 \mathrm{~mL}$ heparin-saline (10 I.U./mL) was flushed through the cannula after intravenous artemether administration or each blood sampling. This reliable model has been routinely used in our laboratory to assess preclinical pharmacokinetics [52-54].

The intravenous pharmacokinetic profiles of artemether were subsequently examined in rats using two different formulations-namely, free artemether solution formulated with cosolvency and artemether-loaded zein nanoparticles. Artemether solution $(5 \mathrm{mg} / \mathrm{mL})$ was prepared in Cremophore EL-saline (1:3) as reported in a previous pharmacokinetic 
study [28], while the dry powder of nanoparticles was reconstituted in isotonic saline to a final artemether concentration of $5 \mathrm{mg} / \mathrm{mL}$. Ten rats were divided into two groups. Group $1(n=5)$ received a single bolus intravenous injection of free artemether solution at $5 \mathrm{mg} / \mathrm{kg}$, while Group $2(n=5)$ received the same dose of artemether in artemether-loaded zein nanoparticles. Serial blood samples were collected from both groups before dosing and at 5, 15, 30, 60, 90, 120, 180, 300, 420, 600 and 1440 min after intravenous injection. To enhance the stability of artemether, $1 \% v / v$ of $0.4 \mathrm{M}$ potassium dichromate was added to heparinized tubes that would be used to collect rat blood samples [55]. After centrifugation at $5000 \times \mathrm{g}$ for $5 \mathrm{~min}$, the plasma samples were harvested and stored at $-40{ }^{\circ} \mathrm{C}$ until liquid chromatography-tandem mass spectrometry (LC-MS/MS) analysis.

\subsection{LC-MS/MS Analysis}

The LC-MS / MS system, consisting of an Agilent 1290 Infinity Liquid Chromatography system (Agilent Technologies, Santa Clara, CA, USA) and an ABSciex QTRAP 5500 mass spectroscopy (AB Sciex, Framingham, MA, USA) equipped with TurboIon Spray probe (AB Sciex), was applied to quantify the plasma level of artemether. Analyst 1.6.2 software (AB Sciex) was used for the operation of the LC-MS/MS system and for data analyses. Nitrogen was used as nebulizing, curtain and collision gases. The ion source was operated in the positive mode. Mass spectrometer parameters including curtain gas, gas 1, gas 2 pressures were set at 20, 40 and $40 \mathrm{psi}$, respectively. Temperature and ion spray voltage were set at $600{ }^{\circ} \mathrm{C}$ and $5500 \mathrm{~V}$, respectively. The precursor-to-product ion transition for artemether was $m / z 316 \rightarrow 267$ and for artemisinin (internal standard) this was $m / z 300$ $\rightarrow 135$ [56]. Optimal compound parameters, namely the declustering potential, entrance potential, collision energy and collision exit cell potential, obtained were 46.00, 10.00, 12.00 and 22.00, respectively, for $m / z 316 \rightarrow 267$ and 85.00, 12.00, 34.58 and 24.00, respectively, for $m / z 300 \rightarrow 135$.

Chromatographic separations were performed using a reversed-phase column (Agilent Poroshell 120 EC-C18: $75 \times 3.0 \mathrm{~mm}, 2.7 \mu \mathrm{m}$ ) with guard column (Agilent Poroshell $120 \mathrm{EC}-\mathrm{C} 18: 5 \times 3.0 \mathrm{~mm}, 2.7 \mu \mathrm{m})$. Acetonitrile was used as the organic phase and aqueous solution containing $0.04 \mathrm{M}$ ammonium acetate and $0.1 \% v / v$ formic acid was used as the aqueous phase. Gradient delivery of the mobile phase at a flow rate of $0.35 \mathrm{~mL} / \mathrm{min}$ at room temperature $\left(\sim 24{ }^{\circ} \mathrm{C}\right)$ was used for the chromatographic separation. The gradient schedule used was (i) $0.00-0.50 \mathrm{~min}$, acetonitrile $50 \%$; (ii) $0.50-6.50 \mathrm{~min}$, acetonitrile $50 \rightarrow$ 95\%; (iii) 6.50-6.60 $\mathrm{min}$, acetonitrile $95 \rightarrow 50 \%$; (iv) 6.60-8.00 $\mathrm{min}$, acetonitrile $50 \%$. MS was operated in multiple reaction monitoring (MRM) mode at a unit mass resolution with a dwell time of $100 \mathrm{~ms}$. The calibration curve ranged from 10 to $1000 \mathrm{ng} / \mathrm{mL}$ artemether $(0.017-3.356 \mathrm{nM})$.

A protein precipitation procedure was employed to clean plasma and extract artemether $[57,58]$. Internal standard working solution was prepared by diluting stock solution of artemisinin with acetonitrile and then spiking with formic acid to obtain $1000 \mathrm{ng} / \mathrm{mL}$ artemisinin solution with $1 \% v / v$ formic acid. During sample preparation, three volumes of internal standard working solution were then added to one volume of rat plasma, vortexed, and centrifuged at $10,000 \times g$ for $10 \mathrm{~min}$ at $4{ }^{\circ} \mathrm{C}$. The supernatant was put into a $250 \mu \mathrm{L}$ plastic autosampler vial (Agilent Technologies) for analysis. For each assay, $10 \mu \mathrm{L}$ of sample was injected into the LC-MS/MS system.

\subsection{Pharmacokinetic Calculation}

All pharmacokinetic analyses were carried out using WinNonlin standard version 1.0 (Scientific Consulting Inc., Apex, NC, USA). Since the log plasma artemether concentrationtime curves of all rats receiving intravenous administration displayed biexponential decline, a classical two-compartment first-order open model was used to represent the intravenous pharmacokinetic profile and the apparent volume of distribution of the central compartment $\left(V_{c}\right)$ was subsequently calculated $[29,30]$. The plasma exposure (area under the plasma concentration-time curve from 0 min to last measurable point (AUC)) was calculated by 
the log trapezoidal method. Clearance $(C L)$, mean residence time $(M R T)$ and terminal elimination half-life $\left(t_{1 / 2} \lambda z\right)$ were also calculated with a noncompartmental method [29,30]. A weighting factor of $1 / y^{2}$ was adopted in all pharmacokinetic modeling [59].

\subsection{Statistics}

All experiments were performed in triplicate unless otherwise stated. The results are expressed as mean \pm standard deviation (SD). The difference between means of two groups were analyzed by two-tailed unpaired $t$-test. Statistically significant differences were indicated by $p$ values less than 0.05 .

Author Contributions: Conceptualization, Y.B.-M., Y.D., H.-S.L. and W.K.N.; methodology, Y.B.-M., Y.D. and H.-S.L.; formal analysis, Y.B.-M., Y.D. and H.-S.L.; investigation, Y.B.-M. and H.-S.L.; resources, H.-S.L. and W.K.N.; data curation, Y.B.-M. and H-.S.L.; writing-original draft preparation, Y.B.-M. and H.-S.L.; writing-review and editing, Y.B.-M., Y.D., H.-S.L. and W.K.N.; supervision, Y.D., H.-S.L. and W.K.N.; project administration, H.-S.L. and W.K.N.; funding acquisition, H.-S.L. and W.K.N. All authors have read and agreed to the published version of the manuscript.

Funding: This research was funded by an Academic Research Fund Tier 1 grant from the Ministry of Education, Singapore (R-148-000-215-112). Y.B.-M. was a recipient of the Singapore International Graduate Award. The APC was funded by H.-S.L.'s personal resources.

Institutional Review Board Statement: This pharmacokinetic study was conducted with strict adherence to the Guidelines on the Care and Use of Animals for Scientific Purposes (Singapore). The study design and animal handling procedures were reviewed and approved by the Institutional Animal Care and Use Committee of the National University of Singapore (NUS) (Project No.: R151273, 05 Nov 2015).

Informed Consent Statement: Not applicable.

Data Availability Statement: The data presented in this study are available on request from the corresponding authors.

Acknowledgments: Y.B.-M., Y.D. and W.K.N. would like to thank the technical staff from the Institute of Chemical and Engineering Sciences, Agency for Science, Technology and Research, Singapore for their technical support. Y.B.-M. and H.-S.L. would like to thank Yu Dai, Feng Liu and Mai Gamal Elhennawy for their help in LC-MS/MS analysis and pharmacokinetic study.

Conflicts of Interest: The authors declare no conflict of interest.

$\begin{array}{ll}\text { Abbreviations } \\ \text { CL } & \text { Clearance } \\ \text { DMSO } & \text { Dimethyl sulfoxide } \\ \text { DSC } & \text { Differential scanning calorimetry } \\ \text { FTIR } & \text { Fourier transform infrared } \\ \text { HPLC } & \text { High performance liquid chromatography } \\ \text { LC-MS/MS } & \text { Liquid chromatography-tandem mass spectrometry } \\ \text { MRT } & \text { Mean residence time } \\ \text { nm } & \text { nanometer } \\ \text { PBS } & \text { Phosphate buffer solution } \\ \text { PDI } & \text { Polydispersity index } \\ \text { pI } & \text { Isoelectric point } \\ \text { RBCs } & \text { Red blood cells } \\ \text { SD } & \text { Standard deviation } \\ t_{1 / 2 \lambda Z} & \text { Terminal elimination half-life } \\ V_{c} & \text { Apparent volume of distribution of the central compartment } \\ \text { WHO } & \text { World Health Organization }\end{array}$




\section{References}

1. Ashley, E.A.; Pyae Phyo, A.; Woodrow, C.J. Malaria. Lancet 2018, 391, 1608-1621. [CrossRef]

2. White, N.J.; Pukrittayakamee, S.; Hien, T.T.; Faiz, M.A.; Mokuolu, O.A.; Dondorp, A.M. Malaria. Lancet 2014, $383,723-735$. [CrossRef]

3. WHO. World Malaria Report 2020: 20 Years of Global Progress and Challenges; World Health Organization: Geneva, Switzerlands, 2020.

4. Gallup, J.L.; Sachs, J.D. The economic burden of malaria. Am. J. Trop. Med. Hyg. 2001, 64, 85-96. [CrossRef]

5. Sachs, J.; Malaney, P. The economic and social burden of malaria. Nature 2002, 415, 680-685. [CrossRef]

6. Septembre-Malaterre, A.; Lalarizo Rakoto, M.; Marodon, C.; Bedoui, Y.; Nakab, J.; Simon, E.; Hoarau, L.; Savriama, S.; Strasberg, D.; Guiraud, P.; et al. Artemisia annua, a Traditional Plant Brought to Light. Int. J. Mol. Sci. 2020, 21, 4986. [CrossRef]

7. WHO. Guidelines for the Treatment of Malaria-Third Edition; World Health Organization: Geneva, Switzerland, 2015.

8. Esu, E.B.; Effa, E.E.; Opie, O.N.; Meremikwu, M.M. Artemether for severe malaria. Cochrane Database Syst. Rev. 2019, 6, CD010678. [CrossRef]

9. Hawkins, M.J.; Soon-Shiong, P.; Desai, N. Protein nanoparticles as drug carriers in clinical medicine. Adv. Drug Deliv. Rev. 2008, 60, 876-885. [CrossRef]

10. Boateng-Marfo, Y.; Dong, Y.C.; Loh, Z.H.; Lin, H.S.; Ng, W.K. Intravenous human serum albumin (HSA)-bound artemether nanoparticles for treatment of severe malaria. Colloids Surf. A Physicochem. Eng. Asp. 2018, 536, 20-29. [CrossRef]

11. Rehman, K.; Lotsch, F.; Kremsner, P.G.; Ramharter, M. Haemolysis associated with the treatment of malaria with artemisinin derivatives: A systematic review of current evidence. Int. J. Infect. Dis. 2014, 29, 268-273. [CrossRef]

12. Paliwal, R.; Palakurthi, S. Zein in controlled drug delivery and tissue engineering. J. Control. Release 2014, 189, 108-122. [CrossRef]

13. Gough, C.R.; Bessette, K.; Xue, Y.; Mou, X.; Hu, X. Air-Jet Spun Corn Zein Nanofibers and Thin Films with Topical Drug for Medical Applications. Int. J. Mol. Sci. 2020, 21, 5780. [CrossRef]

14. Li, K.-K.; Yin, S.-W.; Yin, Y.-C.; Tang, C.-H.; Yang, X.-Q.; Wen, S.-H. Preparation of water-soluble antimicrobial zein nanoparticles by a modified antisolvent approach and their characterization. J. Food. Eng. 2013, 119, 343-352. [CrossRef]

15. Luo, Y.; Teng, Z.; Wang, T.T.; Wang, Q. Cellular uptake and transport of zein nanoparticles: Effects of sodium caseinate. J. Agric. Food Chem. 2013, 61, 7621-7629. [CrossRef]

16. Patel, A.R.; Bouwens, E.C.; Velikov, K.P. Sodium caseinate stabilized zein colloidal particles. J. Agric. Food Chem. 2010, 58, 12497-12503. [CrossRef]

17. Dickinson, E. Properties of emulsions stabilized with milk proteins: Overview of some recent developments. J. Dairy Sci. 1997, 80, 2607-2619. [CrossRef]

18. Shukla, R.; Cheryan, M. Zein: The industrial protein from corn. Ind. Crop Prod. 2001, 13, 171-192. [CrossRef]

19. Strange, E.; Holsinger, V.; Kleyn, D. Chemical properties of thiolated and succinylated caseins. J. Agric. Food Chem. 1993, 41, 30-36. [CrossRef]

20. Podaralla, S.; Perumal, O. Influence of formulation factors on the preparation of zein nanoparticles. AAPS PharmSciTech 2012, 13, 919-927. [CrossRef]

21. Zhong, Q.; Jin, M. Zein nanoparticles produced by liquid-liquid dispersion. Food Hydrocoll. 2018, 23, 2380-2387. [CrossRef]

22. Dong, F.; Padua, G.W.; Wang, Y. Controlled formation of hydrophobic surfaces by self-assembly of an amphiphilic natural protein from aqueous solutions. Soft Matter 2013, 9, 5933-5941. [CrossRef]

23. Ansari, M.T.; Hussain, A.; Nadeem, S.; Majeed, H.; Saeed-Ul-Hassan, S.; Tariq, I.; Mahmood, Q.; Khan, A.K.; Murtaza, G. Preparation and characterization of solid dispersions of artemether by freeze-dried method. BioMed Res. Int. 2015, 2015, 109563. [CrossRef]

24. Lai, L.; Guo, H. Preparation of new 5-fluorouracil-loaded zein nanoparticles for liver targeting. Int. J. Pharm. 2011, 404, 317-323. [CrossRef]

25. Colloidal Dynamics Inc. The Zeta Potential. In Electroacoustic Tutorials; Colloidal Dynamics Inc.: Ponte Vedra Beach, FL, USA, 1999; pp. 1-4.

26. Ibrahim, N.; Ibrahim, H.; Dormoi, J.; Briolant, S.; Pradines, B.; Moreno, A.; Mazier, D.; Legrand, P.; Nepveu, F. Albumin-bound nanoparticles of practically water-insoluble antimalarial lead greatly enhance its efficacy. Int. J. Pharm. 2014, 464, 214-224. [CrossRef]

27. Joshi, M.; Pathak, S.; Sharma, S.; Patravale, V. Design and in vivo pharmacodynamic evaluation of nanostructured lipid carriers for parenteral delivery of artemether: Nanoject. Int. J. Pharm. 2008, 364, 119-126. [CrossRef]

28. Li, Q.G.; Peggins, J.O.; Fleckenstein, L.L.; Masonic, K.; Heiffer, M.H.; Brewer, T.G. The pharmacokinetics and bioavailability of dihydroartemisinin, arteether, artemether, artesunic acid and artelinic acid in rats. J. Pharm. Pharmacol. 1998, 50, 173-182. [CrossRef]

29. Lin, H.S.; Ho, P.C. Preclinical pharmacokinetic evaluation of resveratrol trimethyl ether in Sprague-Dawley rats: The impacts of aqueous solubility, dose escalation, food and repeated dosing on oral bioavailability. J. Pharm. Sci. 2011, 100, 4491-4500. [CrossRef]

30. Yeo, S.C.; Ho, P.C.; Lin, H.S. Pharmacokinetics of pterostilbene in Sprague-Dawley rats: The impacts of aqueous solubility, fasting, dose escalation, and dosing route on bioavailability. Mol. Nutr. Food Res. 2013, 57, 1015-1025. [CrossRef] 
31. German, P.I.; Aweeka, F.T. Clinical pharmacology of artemisinin-based combination therapies. Clin. Pharmacokinet. 2008, 47, 91-102.

32. Medhi, B.; Patyar, S.; Rao, R.S.; Byrav, D.S.P.; Prakash, A. Pharmacokinetic and toxicological profile of artemisinin compounds: An update. Pharmacology 2009, 84, 323-332. [CrossRef]

33. Morris, C.A.; Duparc, S.; Borghini-Fuhrer, I.; Jung, D.; Shin, C.S.; Fleckenstein, L. Review of the clinical pharmacokinetics of artesunate and its active metabolite dihydroartemisinin following intravenous, intramuscular, oral or rectal administration. Malar. J. 2011, 10, 263. [CrossRef]

34. Garg, A.; Tomar, D.S.; Bhalala, K.; Wahajuddin, M. Development and investigation of Artemether loaded binary solid lipid nanoparticles: Physicochemical characterization and In-Situ single-pass intestinal permeability. J. Drug Deliv. Sci. Technol. 2020, 60, 102072. [CrossRef]

35. Khatri, H.; Chokshi, N.; Rawal, S.; Patel, B.M.; Badanthadka, M.; Patel, M.M. Fabrication and In Vivo evaluation of ligand appended paclitaxel and artemether loaded lipid nanoparticulate systems for the treatment of NSCLC: A nanoparticle assisted combination oncotherapy. Int. J. Pharm. 2020, 583, 119386. [CrossRef]

36. RinTalib, S.; Ahmed, N.; Khan, D.; Khan, G.M.; Ur Rehman, A. Chitosan-chondroitin based artemether loaded nanoparticles for transdermal drug delivery system. J. Drug Deliv. Sci. Technol. 2021, 61, 102281.

37. Pawar, S.; Shende, P. 2(2) factorial design-based biocompatible microneedle arrays containing artemether co-loaded with lumefantrine nanoparticles for transepidermal delivery. Biomed. Microdevices 2020, 22, 19. [CrossRef]

38. Souza, A.C.M.; Mosqueira, V.C.F.; Silveira, A.P.A.; Antunes, L.R.; Richard, S.; Guimaraes, H.N.; Grabe-Guimaraes, A. Reduced cardiotoxicity and increased oral efficacy of artemether polymeric nanocapsules in Plasmodium berghei-infected mice. Parasitology 2018, 145, 1075-1083. [CrossRef]

39. Parashar, D.; Aditya, N.P.; Murthy, R.S. Development of artemether and lumefantrine co-loaded nanostructured lipid carriers: Physicochemical characterization and In Vivo antimalarial activity. Drug Deliv. 2016, 23, 123-129. [CrossRef]

40. Sidhaye, A.A.; Bhuran, K.C.; Zambare, S.; Abubaker, M.; Nirmalan, N.; Singh, K.K. Bio-inspired artemether-loaded human serum albumin nanoparticles for effective control of malaria-infected erythrocytes. Nanomedicine 2016, 11, 2809-2828. [CrossRef]

41. Jain, K.; Sood, S.; Gowthamarajan, K. Optimization of artemether-loaded NLC for intranasal delivery using central composite design. Drug Deliv. 2015, 22, 940-954. [CrossRef]

42. Eltayeb, S.E.; Su, Z.; Shi, Y.; Li, S.; Xiao, Y.; Ping, Q. Preparation and optimization of transferrin-modified-artemether lipid nanospheres based on the orthogonal design of emulsion formulation and physically electrostatic adsorption. Int. J. Pharm. 2013, 452, 321-332. [CrossRef]

43. Aditya, N.P.; Patankar, S.; Madhusudhan, B.; Murthy, R.S.; Souto, E.B. Arthemeter-loaded lipid nanoparticles produced by modified thin-film hydration: Pharmacokinetics, toxicological and In Vivo anti-malarial activity. Eur. J. Pharm. Sci. 2010, 40, 448-455. [CrossRef]

44. Sun, D.; Zhou, S.; Gao, W. What Went Wrong with Anticancer Nanomedicine Design and How to Make It Right. ACS Nano 2020, 14, 12281-12290. [CrossRef]

45. Alhakamy, N.A.; Ahmed, O.A.A.; Aldawsari, H.M.; Alfaifi, M.Y.; Eid, B.G.; Abdel-Naim, A.B.; Fahmy, U.A. Encapsulation of Lovastatin in Zein Nanoparticles Exhibits Enhanced Apoptotic Activity in HepG2 Cells. Int. J. Mol. Sci. 2019, 20, 5788. [CrossRef]

46. Algandaby, M.M.; Al-Sawahli, M.M.; Ahmed, O.A.A.; Fahmy, U.A.; Abdallah, H.M.; Hattori, M.; Ashour, O.M.; Abdel-Naim, A.B Curcumin-Zein Nanospheres Improve Liver Targeting and Antifibrotic Activity of Curcumin in Carbon Tetrachloride-Induced Mice Liver Fibrosis. J. Biomed. Nanotechnol. 2016, 12, 1746-1757. [CrossRef]

47. Cheng, W.; Wang, B.; Zhang, C.; Dong, Q.; Qian, J.; Zha, L.; Chen, W.; Hong, L. Preparation and preliminary pharmacokinetics study of GNA-loaded zein nanoparticles. J. Pharm. Pharmacol. 2019, 71, 1626-1634. [CrossRef]

48. Dokunmu, T.M.; Ahanonu, C.L.; Abegunde, O.O.; Adeyemi, O.A. Artemisinin-induced delayed hemolysis after administration of artesunate and artesunate-amodiaquine in malaria-free Wistar rats. Biomed. Res. Ther. 2017, 4, 1246-1260. [CrossRef]

49. D'Souza, S. A review of in vitro drug release test methods for nano-sized dosage forms. Adv. Pharmaceut. 2014, $2014,304757$.

50. Chen, W.; Yeo, S.C.; Elhennawy, M.G.; Xiang, X.; Lin, H.S. Determination of naturally occurring resveratrol analog trans-4,4'dihydroxystilbene in rat plasma by liquid chromatography-tandem mass spectrometry: Application to a pharmacokinetic study. Anal. Bioanal. Chem. 2015, 407, 5793-5801. [CrossRef]

51. Yeo, S.C.M.; Fenwick, P.S.; Barnes, P.J.; Lin, H.S.; Donnelly, L.E. Isorhapontigenin, a bioavailable dietary polyphenol, suppresses airway epithelial cell inflammation through a corticosteroid-independent mechanism. Br. J. Pharmacol. 2017, 174, 2043-2059. [CrossRef]

52. Dai, Y.; Yeo, S.C.M.; Barnes, P.J.; Donnelly, L.E.; Loo, L.C.; Lin, H.S. Pre-clinical Pharmacokinetic and Metabolomic Analyses of Isorhapontigenin, a Dietary Resveratrol Derivative. Front. Pharmacol. 2018, 9, 753. [CrossRef]

53. Dai, Y.; Lim, J.X.; Yeo, S.C.M.; Xiang, X.; Tan, K.S.; Fu, J.H.; Huang, L.; Lin, H.S. Biotransformation of Piceatannol, a Dietary Resveratrol Derivative: Promises to Human Health. Mol. Nutr. Food Res. 2020, 64, e1900905. [CrossRef]

54. Chen, W.; Yeo, S.C.M.; Elhennawy, M.; Lin, H.S. Oxyresveratrol: A bioavailable dietary polyphenol. J. Funct. Foods 2016, 22, 122-131. [CrossRef]

55. Lindegardh, N.; Hanpithakpong, W.; Kamanikom, B.; Pattayaso, J.; Singhasivanon, P.; White, N.J.; Day, N.P. Quantification of dihydroartemisinin, artesunate and artemisinin in human blood: Overcoming the technical challenge of protecting the peroxide bridge. Bioanalysis 2011, 3, 1613-1624. [CrossRef] 
56. Huang, L.; Jayewardene, A.L.; Li, X.; Marzan, F.; Lizak, P.S.; Aweeka, F.T. Development and validation of a high-performance liquid chromatography/tandem mass spectrometry method for the determination of artemether and its active metabolite dihydroartemisinin in human plasma. J. Pharm. Biomed. Anal. 2009, 50, 959-965. [CrossRef]

57. Elhennawy, M.G.; Lin, H.S. Determination of Tangeretin in Rat Plasma: Assessment of Its Clearance and Absolute Oral Bioavailability. Pharmaceutics 2018, 10, 3. [CrossRef]

58. Elhennawy, M.G.; Lin, H.S. Dose- and time-dependent pharmacokinetics of apigenin trimethyl ether. Eur. J. Pharm. Sci. 2018, 118, 96-102. [CrossRef]

59. Yeo, S.C.; Sviripa, V.M.; Huang, M.; Kril, L.; Watt, D.S.; Liu, C.; Lin, H.S. Analysis of trans-2,6-difluoro-4'-(N,Ndimethylamino)stilbene (DFS) in biological samples by liquid chromatography-tandem mass spectrometry: Metabolite identification and pharmacokinetics. Anal. Bioanal. Chem. 2015, 407, 7319-7332. [CrossRef] 